\title{
The indoleamine 2,3 dioxygenase pathway drives intratumoral B cell
}

\section{maintenance.}

\author{
by
}

Burles A. Johnson III ${ }^{1,2,3}$, Adam K. Aragaki², Donna M. Williams³, Ophelia Rogers ${ }^{3}$, Jack Mountain², Li Luo ${ }^{3}$, Wenhao Zhang ${ }^{3}$, Lingling Xian³ ${ }^{3}$ Mingxiao Feng ${ }^{2}$, Lionel Chia ${ }^{3}$, Dominic

Dordai $^{4}$, Noah M. Hahn ${ }^{1,2}$, Stephen Desiderio ${ }^{4}$, Theodore S. Johnson ${ }^{5}$, David J. McConkey², and Linda M.S. Resar ${ }^{1,3,6}$.

${ }^{1}$ Department of Oncology, Johns Hopkins University School of Medicine, Baltimore, MD 21205

2Johns Hopkins Greenberg Bladder Cancer Institute, Johns Hopkins University School of Medicine, 600 North Wolfe Street, Park 219, Baltimore, MD 21287

${ }^{3}$ Division of Hematology, Department of Medicine, Johns Hopkins University School of Medicine, 720 Rutland Avenue, Ross Research Building, Room 1025, Baltimore, MD 21205

${ }^{4}$ Institute for Basic Biomedical Sciences, Johns Hopkins University School of Medicine, Baltimore, MD 21205

${ }^{5}$ Georgia Cancer Center and Department of Pediatrics, Augusta University, $112015^{\text {th }}$ Street, Augusta, GA 30912

${ }^{6}$ Department of Pathology and Institute for Cellular Engineering, Johns Hopkins University School of Medicine, Baltimore, MD 21205

Abstract: 219 words; Manuscript Text: 4929 words

Correspondence: bjohn117@jhmi.edu or Iresar@jhmi.edu

Abbreviations: IDO1, indoleamine 2,3 dioxygenase-1; Bregs, regulatory B cells, Tregs, regulatory T cells; LLC, Lewis Lung Carcinoma; PD-1/L, programmed cell death protein-1/ligand; APCs, antigen presenting cells; MDSCs, myeloid derived suppressor cells; IL, interleukin. 


\section{Abstract (219 words)}

B cells have been implicated as central regulators of immune responses in settings as diverse as mammalian pregnancy, mucosal tolerance, chronic infection states, autoimmunity, and the tumor microenvironment. Despite the established importance of $B$ cells in these environments, the mechanisms by which B cells are maintained in these contexts remain undefined. Here, we report that IDO1 pathway inhibition with D-1-methyl-tryptophan (D-1MT) and linrodostat significantly decreases tumor infiltrating B (TIL-B) cells in a preclinical model of melanoma. Single cell RNA sequencing (scRNAseq) of murine melanoma demonstrate TIL-B cells are heterogeneous but primarily express markers consistent with an immune stimulatory phenotype. D-1MT decreases splenic B cells and bone marrow derived B cell precursors in tumor-bearing mice, suggesting that IDO1 pathway inhibition impedes B cell maturation. D-1MT decreases intratumoral myeloid derived suppressor cells (MDSCs), which are essential for maintenance of TIL-B cells. Unlike D-1MT, genetic deletion of tumor Ido1 does not impact TIL-B or MDSC numbers. In human solid tumors, intratumoral IDO1 expression consistently associates with high expression of a pan-B cell gene signature, and in patients with melanoma, scRNAseq analysis of tumor samples revealed most TIL-B cells express IDO1. Collectively, our data reveal the impact of pharmacologic IDO1 inhibition on B cells, which may have therapeutic implications for patients with solid tumors by informing the design of future oncology clinical trials. 


\section{Introduction}

B cells are a critical regulator of the immune response in settings such as pregnancy ${ }^{1}$, infection $^{2}$, autoimmunity ${ }^{3}$, and cancer ${ }^{4}$. As B cells have diverse function such as antigen presentation, T cell activation, immunoglobulin generation, and cytokine production, these cells can exude powerful context dependent immune stimulatory or immune inhibitory effects ${ }^{1-4}$. In cancer, B cells can stimulate $\mathrm{T}$ cell responses to enhance anti-tumor immunity ${ }^{5}$; however, immune-regulatory B cells (Bregs) suppress responses ${ }^{6,7}$ and correlate with advanced tumor stage, disease progression, and/or decreased survival in diverse solid tumors ${ }^{8-13}$. An understanding of mechanisms governing intratumoral B cell maintenance may provide additional therapeutic targets to enhance stimulatory B cell function, and/or mitigate suppressive Breg effects.

Accumulating evidence suggests amino acid metabolism is critical for B cell function and survival in certain conditions ${ }^{14,15}$. While much focus has centered on glutamine metabolism in regulating $B$ cell function ${ }^{16}$, other amino acid pathways can modulate $B$ cells. For example, $B$ cell specific expression of the tryptophan catabolizing enzyme indoleamine 2,3 dioxygenase-1 (IDO1) suppresses B cell proliferation and antibody secretion in response to T cell-independent antigen stimulation ${ }^{17}$. Conversely, IDO1 activity facilitates autoantibody production in a model of gastric dysplasia $^{18}$. Thus, the role of IDO1 in B cells is likely complex and context dependent. B cells play an important role in regulating the immune response and therapeutic efficacy in cancer ${ }^{19}$, and IDO1 mediates immune suppression in the tumor microenvironment ${ }^{20}$. This study assesses the relationship between IDO1 and B cells in preclinical cancer models.

\section{Materials and Methods}

\section{Mice and cell lines}

C57BL/6 mice (The Jackson Laboratory) were housed in a pathogen-free environment, and experimental protocols were approved by the Johns Hopkins Institutional Animal Care and 
Use Committee. Similar to others studying the role of IDO1 in cancer ${ }^{21,22}$, female mice were used. We used young adult (ages 2-6 months) mice that were matched for age within each experiment. Experiments were performed at least twice with three to nine mice per group per experiment, and data from each experimental replicate were pooled for final analyses. The exception was Fig. 1G which was performed once with seven mice per group.

B16F10 mouse melanoma ${ }^{23-25}$ and Lewis Lung Carcinoma (LLC) ${ }^{21,26}$ were cultured in RPMI 1640 media with 10\% Fetal Bovine Serum (FBS). All cell lines were propagated in flasks at $37^{\circ} \mathrm{C}$ and $5 \% \mathrm{CO}_{2}$. By STR authentication on November $6,2019, \mathrm{~B} 16 \mathrm{~F} 10$ and LLC cell lines were found to match the established genetic profile.

\section{Tumor Injections and D-1-methyl-tryptophan}

For solid tumor models, we injected $5 \times 10^{4}$ B16F10 cells or $1 \times 10^{5}$ LLC cells (diluted in sterile PBS) subcutaneously into the lower left mouse thigh (considered day 0). For experiments with flow cytometry, the IDO1 pathway inhibitor D-1-methyl-tryptophan (D-1MT or indoximod, $2 \mathrm{mg} / \mathrm{mL}$, Sigma) or control saccharin water was given to mice in bottles protected from light beginning on day 8 , with tumor, spleen, and/or bone marrow harvest on day 13 . For experiments with the direct IDO1 inhibitor linrodostat (BMS-986205, Selleck Chemicals), mice were treated with vehicle $(200 \mu \mathrm{L}$ of 40 proof ethanol), or $100 \mathrm{mg} / \mathrm{kg}$ linrodostat (diluted in sterile water from a stock solution of 200 proof ethanol to 40 proof ethanol just before gavage) via oral gavage daily. Treatments were started on day 8 , with harvest on day 13.

For experiments involving MDSC depletion, anti-Gr1 antibody (Biolegend, Cat. No. 108453) or isotype control antibody (Biolegend, Cat. No. 400671) was administered (200 $\mu \mathrm{g}$, intraperitoneal) on day 7, 9, and 12 following injection with B16F10 melanoma on day 0 . Mice were harvested on day 13.

For tumor growth experiments, control or D-1MT water was initiated on day 8 . Tumors were measured using calipers every 3-4 days, and tumor volume was calculated using the formula 
$V=L^{*}\left(W^{2}\right)^{*}(\pi / 6)$, where $V$ is the tumor size in $\mathrm{mm}^{3}, L$ is the longest diameter, and $W$ is the shortest diameter $^{27}$. Mice were weighed at baseline and with each tumor measurement.

\section{Preparation for Tumor Infiltrating Lymphocyte Analysis}

After mouse sacrifice on day 13 (or day 20 in Fig. 1B as noted), tumors were isolated and weighed. Each tumor was placed in a separate well in a 6 well plate in RPMI media supplemented with $10 \%$ FBS, 1000 units/mL collagenase, and $0.05 \mathrm{mM} \beta$-mercaptoethanol. Tumors were dissected manually, followed by incubation for 1 hour at $37^{\circ} \mathrm{C}$. Cells were filtered through a 70 $\mu \mathrm{m}$ cell strainer and resuspended to a total concentration of $2 \times 10^{7} / \mathrm{mL}$ before staining. For spleen and bone marrow analysis, red cells were lysed with ACK (Ammonium-Chloride-Potassium) Lysis Buffer (ThermoFisher) before counting and staining.

\section{Analytical Flow Cytometry}

We used the following antibodies for cell surface staining: CD19 (clone 6D5), Gr-1 (RB68C5), IgD (11-26c.2a), PD-1 (29F.1A12), PD-L1 (10F.9G2), PD-L2 (TY25), CD21/35 (7E9), CD23 (B3B4), CD24 (30-F1), Tim1 (RMT1-4), CD5 (53-7.3), and CD25 (PC61; all from Biolegend), and CD45 (clone 30-F11), CD19 (1D3), CD11b (M1/70), B220 (RA3-6B2), IgM (R6-60.2), and CD1d (1B1; all from BD Biosciences). Cells were first incubated with CD16/CD32 Fc block (BD Biosciences), followed by a cell viability stain (either Live/Dead Fixable Aqua Dead Cell Stain Kit, 405 nm excitation, Invitrogen ref. no. L34966; or Fixable Viability Stain 700; BD Horizon Cat. No. 564997). For tumor cell analysis, controls containing only viability stain were used to set initial gating structure ${ }_{\bar{j}}$ to exclude dead cells and doublets (Sup. Fig. 1). Fluorescence minus one controls were used as in Sup. Fig. 1, to determine cells staining positive for a particular fluorophore above background, as described ${ }^{28,29}$. Leukocytes were distinguished from tumor cells by CD45 staining. The LSR II flow cytometer or FACSCelesta (BD Biosciences) was used for cell acquisition, and Diva software was used for analysis.

\section{RNA Sequencing Analyses}


All code for data acquisition, preprocessing, analysis, and figure generation is available on GitHub. TCGA bulk RNA sequencing data for muscle invasive bladder cancer, lung adenocarcinoma, squamous cell lung carcinoma, and melanoma were downloaded from the Genomic Data Commons using the GenomicDataCommons package ${ }^{30}$. Single cell RNA sequencing (scRNAseq) data for implanted B16F10 tumors are publicly available ${ }^{31}$ and were downloaded from ArrayExpress: E-MTAB-7427.

For pre-processing of scRNAseq data, TPM (transcripts-per-million) expression was downloaded, data were pre-filtered, and expression was scaled by log2(TPM + 1). TCGA data were normalized and scaled using DESeq2's vst (variance stabilizing transformation) ${ }^{32}$.

For data analysis, GSVA ${ }^{33}$ (geneset variation analysis) was performed using a Gaussian kernel estimation of the cumulative distribution function. Scores were calculated using the mx.diff mode such that under the null hypothesis the distribution of scores would approximate a normal distribution with mean 0 . Scores that were above 0 were labelled as 'hi', while those below zero were labelled as 'lo'. For gene expression, a cell is ' + ' for a gene if the expression cutoff is $>0$ but 'hi' if the expression of a gene is obviously bimodal, setting the cutoff between low/no expression and high expression. $R$ Version 4.0.3 was used for all scRNAseq and bulk RNA sequencing analyses ${ }^{34}$.

\section{IDO-specific siRNA expression vectors}

Ido1 shRNAs targeting different specific sequences of Ido1 (shRNA-Ido1-908 and shRNA-Ido1909) expressed by a lentiviral vector were designed by the Broad Institute (Cambridge, MA, US) and purchased from MilliporeSigma (Cat No. SHCLNG-NM_008324; clone ID: TRCN0000066908; clone ID: TRCN0000066909). The empty shRNA vector, pLKO.1 (TRC) (MilliporeSigma, Cat No. SHC001) was used as a control. 


\section{Lentivirus Production}

For lentivirus production ${ }^{35,36}$, COS1 African green monkey kidney epithelial cells (American Type Culture Collection, Cat. No. ATCC ${ }^{\circledR}$ CRL $1650^{\mathrm{TM}}$ ) were transfected with pCMV- $\Delta$ R8.91 (Creative Biogene, Cat. No. OVT2971) and pCMV-VSVg packaging plasmids (Addgene, Cat. No. 8454) and the Ido1 shRNA using Lipofectamine 2000 (ThermoFisher Scientific, Cat. No. 11668027). Cells were transfected in virus collection medium (DMEM supplemented with $1 \%$ FBS) which was replaced with fresh medium $6 \mathrm{~h}$ after transfection. The culture supernatants were collected at $24 \mathrm{~h}$, $48 \mathrm{~h}$ and $72 \mathrm{~h}$ after transfection, filtered, and concentrated.

\section{Generation of B16F10 ID01 silenced stable cell lines}

B16F10 cells were transduced using standard lentivirus transduction protocols (see Addgene protocol found at: http://www.addgene.org/tools/protocols/plko/\#E). Briefly, B16F10 cells were seeded into 24-well plates and grown to $\sim 80 \%$ confluence then each of the 3 viruses (TRC, shRNA-Ido1-908 and shRNA-Ido1-909) plus a mock control were added in triplicate wells in complete medium (DMEM, 10\% FBS) supplemented with $100 \mathrm{U} / \mathrm{ml}$ penicillin, $100 \mathrm{ug} / \mathrm{ml}$ streptomycin (ThermoFisher Scientific, Cat. No. 15140-122) plus 8 ug/ml polybrene transfection reagent (MilliporeSigma, Cat. No. TR-1003-G). Cells were incubated at $4-8^{\circ} \mathrm{C}$ for two hours then placed in a $37^{\circ} \mathrm{C}$ incubator overnight. Cells were then split into 6 -well plates in complete medium and incubated for two days to allow recovery before placing under $2 \mathrm{ug} / \mathrm{ml}$ puromycin selection (MilliporeSigma, Cat. No. P8833-25MG).

\section{Analysis of Ido1 silencing in transduced B16F10}

B16F10 cells normally have very low levels of Ido1 expression. Therefore, in order to test the potency and efficacy of the Ido1 silencing, we induced $/ d o 1$ by incubating $200 \mathrm{U} / \mathrm{ml}$ recombinant murine IFN-y (PeproTech, Cat. No. 315-05) at $37^{\circ} \mathrm{C}$ for 5 hours prior to harvesting for RNA expression by qRT-PCR. Total RNA was extracted from the parental and Ido1 silenced B16F10 
cell lines using the Qiashredder (Qiagen, Cat. No. 79654) and RNeasy mini kit (Qiagen, Cat. No. 74104) with on-column RNase-Free DNase digestion (Qiagen, Cat. No.79254). First strand cDNA was generated using the High-Capacity cDNA Reverse Transcription Kit (ThermoFisher Cat. No. 4368814). Real-time PCR was performed on an AriaMx Real-Time PCR system (Agilent) using primer/probes from ThermoFisher targeting exon 5 of the Ido1 gene (Mm00492590_m1) and normalized to beta-actin (P/N 4352933E). Amplification was performed using TaqMan Universal PCR Master Mix (ThermoFisher Scientific, Cat. No. 4324018) and analyzed using the ${ }^{\Delta \Delta} \mathrm{Ct}$ method $^{37}$.

\section{Statistics}

For mouse experiments, data from each experimental group was tested for normality by the RyanJoiner Test ${ }^{38}$ and D'Agostino-Pearson Test ${ }^{39}$. The student's t-test was used if data were normally distributed; the Mann-Whitney test was used otherwise ${ }^{40}$. $P$ values $\leq 0.05$ were considered significant. For scRNAseq and bulk RNA sequencing analyses, testing for equivalence of means was performed using a two-tailed Welch's unequal variances t-test, with a significance cutoff of $p$ $<0.05$. As only a single comparison was made for each dataset, no multiple testing was applied.

\section{Results}

\section{ID01 pathway inhibition decreases tumor infiltrating B cells}

To explore the role of the IDO1 pathway in antitumor immunity, we used D-1-methyltryptophan (D-1MT, indoximod), a tryptophan mimetic with established pharmacodynamics in humans and mice ${ }^{41,42}$, in the well-established implantable B16F10 model, which is an aggressive melanoma cell line that is wild-type for BRAF. Eight days after implanting B16F10 melanoma tumor cells into flanks of immunocompetent mice (see Fig. 1A) ${ }^{23,24}$, mice were treated with either D-1MT administered in drinking water or control water as previously described ${ }^{47}$. Similar to previous reports, IDO1 pathway inhibition had no significant effect on tumor size within the first 5 
days of therapy (day 13 after implantation), although there was a modest decrease in tumor size after 12 days of therapy (day 20 after tumor cell implantation; Fig. 1B). To define the effects of D-1MT on the immune landscape, we first examined tumor infiltrating leukocytes (TILs) at early stages after tumor implantation (day 5 of therapy) using flow cytometry. There was a modest, but consistent, decrease in TIL frequency and absolute number per gram of tumor tissue (Fig. 1C). To determine whether intra-tumoral T cells changed with D-1MT treatment, we used the pan T cell marker CD3. Similar to prior results in a syngeneic murine model of lung cancer ${ }^{22}$, we found no change in $\mathrm{CD}^{\text {pos }} \mathrm{T}$ cell frequency or absolute number with $\mathrm{D}-1 \mathrm{MT}$ treatment (Fig. 1D).

Because IDO1 regulates $B$ cells in non-cancer models ${ }^{18,48}$, we tested whether D-1MT affected the frequency and/or absolute number of TIL-B cells using the pan B cell marker, CD19. In control, untreated mice, the frequency and number of TIL-B cells is rare, comprising only $\sim 0.1 \%$ of total live cells (Fig. 1E). Strikingly, however, there was a profound decrease in CD19pos TIL-B cell frequency and absolute number per gram tumor in D-1MT treated mice after 5 days of treatment (Fig. 1E), suggesting that D-1MT treatment either decreased the total $B$ cell number and/or expression of CD19 on the B cell surface. To help distinguish between these possibilities, we tested whether TILs expressing other pan-B cell markers were also decreased, reasoning that a reduction would favor a decrease in total B cell number, since it would be less likely for D-1MT to repress expression of multiple B cell markers. To this end, we included B220 (a marker for B cell precursors and mature B cells), IgM (a marker for both immature and mature B cells), and $\lg \mathrm{D}$ (found on late transitional and mature B cells). TIL-B cells expressing these markers decreased following IDO1-inhibitor therapy, consistent with a decrease in B cell frequency and number (Fig. 1F).

To determine whether D-1MT mediated decrease in TIL-B cells was due to an on-target effect of IDO1 inhibition, we treated melanoma bearing mice with the direct IDO1-inhibitor linrodostat. Consistent with the above results, linrodostat treatment significantly decreased TILB cells (Fig. 1G). To determine whether these results are generalizable to another tumor model, 
we tested a murine lung cancer implantation model (LLC $)^{21,26}$. Similar to our findings in melanoma, D-1MT treatment significantly decreased TIL-B cell infiltration in the LLC model (Fig. 1H). Together, these data suggest that IDO1 pathway inhibition decreases the number of TIL-B cells in preclinical models of melanoma and lung cancer.

\section{Melanoma TIL-B cells have a heterogeneous phenotype}

Recent data suggests improved prognosis in melanoma patients with high levels of TIL-B cells $^{49}$, however increases in TIL-B cells also seems to correlate with resistance to BRAF targeted therapy ${ }^{50}$. To more fully understand this dichotomy, we sought to characterize this population further by analyzing TIL-B cells from publicly available single cell RNA sequencing (scRNAseq) data from implanted B16F10 tumors ${ }^{31}$. We found that TIL-B cells were uniformly positive for lghm, and most expressed lghd, suggesting TIL-B cells are naïve or transitional (Fig. 2A, left). Next, we determined that TIL-B cells did not experience class switching, as TIL-B cells did not express $\lg G$ or $\lg A$ heavy chain genes, further indicating that TIL-B cells are naïve or transitional. TIL-B cells also express $\lg M$, IgD, and the pan-B cell marker B220 by flow cytometry (Sup. Fig. 2A). We found that $19 \%$ of TIL-B cells express the transitional B cell specific gene $C d 93^{51}$ (Fig. 2B, left), 44\% of TIL-B cells express the marginal zone specific gene Mzb1 (Fig. 2B, center) and $62 \%$ of TIL-B cells express Cd27 (Fig. 2B, right), an activation marker found on B1 or innate memory cells (CD270os $\left.\operatorname{lgh} D^{\text {pos }}\right)$, or naïve or transitional cells $\left(C D 27^{\text {neg }} / g h D^{\text {pos }}\right)$ (Sup. Fig. 2B).

To define the function of TIL-B cells, we first examined TIL-B cells for expression of genes encoding activation markers and costimulatory molecules. Most TIL-B cells express Ccr7 (Fig. 2C), which is expressed by a TIL-B cell subset that correlates with response to checkpoint immunotherapy in patients with advanced melanoma ${ }^{19}$. A majority of TIL-B cells express genes encoding the activation markers CD69, CD81, and CD86, but fewer TIL-B cells express genes encoding for CD80 (35\%), the costimulatory molecule CD40 (30\%), and the chemokine receptor CXCR5 (46\%), which facilitates B cell receptor mediated activation ${ }^{52}$ (Fig. 2C). 
To examine co-expression of activation markers by TIL-B cells, we graphed Ccr7 and Cd69 along with other activation related genes. Most TIL-B cells are Ccr7 ${ }^{\text {high }}$ Cd69high $(42 \%$ of all TIL-B cells) or Ccr7 $7^{\text {high }}$ Cd69 low/neg (another 40\%) (Fig. 2D, left). About half of Ccr7high $C d 69^{\text {high }}$ and Ccr7high $^{\text {Cd69 }}{ }^{\text {low/neg }}$ TIL-B cells express Cd81, Cd86, and/or Cxcr5 (Fig. 2D, right), suggesting many TIL-B cells are in an activated state. Most TIL-B cells express antigen processing (Fig. 2E) and major histocompatibility complex (MHC) genes (Sup. Fig. 2C), suggesting many TIL-B cells are primed for antigen presentation.

Next, we interrogated the scRNAseq data for expression of genes associated with immune suppression. TIL-B cells do not express genes found in a subset of Bregs called B10 cells ${ }^{53}$ (Cd5 or I/10, Fig. 2F). Moreover, TIL-B cells do not express the IL-35 associated gene //12a, expressed by IL-35 expressing Bregs ${ }^{54}$ (Fig. 2F). However, consistent with previous reports that TGF $\beta$ is produced by a Breg subset ${ }^{55}$, $26 \%$ of TIL-B cells express Tgfb1 (Fig. 2F). Additionally, a subset of TIL-B cells express II2ra (CD25), which identifies a Breg subset ${ }^{56}$ (Fig. 2F).

Finally, we interrogated TIL-B cells for expression of immune checkpoints targeted by commonly used immunotherapeutic agents. Consistent with recent data demonstrating TIL-B cells promote anti-melanoma immunity in response to PD-directed therapy ${ }^{19}$, TIL-B cells express Cd274 (PD-L1), but only minimal Pdcd1 (PD-1) or Pdcd1lg2 (PD-L2) (Fig. 2G). A minority of TILB cells express Lag3, and very few TIL-B cells express the inhibitory costimulatory molecule B7H3 (Cd276, Fig. 2G). However, a significant minority of TIL-B cells express the inhibitory checkpoint Cd200 (Fig. 2G).

To determine whether TIL-B cells co-express immune inhibitory genes, we graphed TILB co-expression of Tgfb1 and Cd200 (Fig. 2H). Very few TIL-B cells co-expressed Tgfb1 and Cd200 (Fig. 2H). As one report suggests Bregs can express Cd69, we graphed Cd69 versus Tgfb1 expression (along with Ccr7) and found a fraction of TIL-B cells ( 15\%) were Tgfb $1^{\text {pos }} \mathrm{Cd} 69^{\text {high }} \mathrm{Ccr} 7^{\text {high }}$ (Fig. 2I). Most TIL-B cells expressing multiple activation markers are 
likely immune stimulatory, however a cohort of TIL-B cells within this subset may have inhibitory function.

In summary, most CD19 ${ }^{\text {pos }}$ TIL-B cells express Ighm and Ighd, and are not class-switched. TIL-B cells are markedly heterogeneous, including transitional and marginal zone phenotypes. While a minority of TIL-B cells express immune suppressive genes, most have an activated phenotype.

\section{ID01 pathway inhibition decreases splenic transitional B cells}

Clinical studies have shown that patients treated with IDO1-inhibitor drugs can develop a transient lymphopenia ${ }^{42}$, and our studies demonstrate D-1MT decreases TIL-B cells in mice. To determine whether the effect of D-1MT on TIL-B cells results from a systemic decrease in B cells, we analyzed spleens from control and D-1MT treated tumor-bearing mice. There was a significant decrease in total splenic B cells in mice with B16F10 tumors (Fig. 3A) and LLC tumors (Fig. 3B) after D-1MT treatment. D-1MT treatment also decreased spleen weights (normalized to mouse weight) in tumor-bearing mice, consistent with the decrease in total intrasplenic B cells (Sup. Fig. 3). Because IDO1 is known to be inducible in a counter-regulatory fashion in settings of inflammation (including tumors) ${ }^{45,57}$, we hypothesized that D-1MT treatment in mice lacking tumors would not significantly reduce splenic B cells, which is the result we observed (Fig. $\mathbf{3 C}$ ).

In order to determine whether D-1MT impacts the same B cell subset within tumors and spleen, we performed additional phenotypic analysis of splenic B cells. To do this, we initially classified splenic CD19pos B cells into marginal zone plus marginal zone precursors $\left(\lg M^{\text {high }} C D 21^{\text {high }}\right)$, transitional $\left(\lg M^{\text {high }} C D 21^{\text {low }}\right)$, and follicular $\left(\lg M^{\text {pos }} C D 21^{\text {pos }}\right)$ subsets. We found that D-1MT decreased splenic transitional B cells, but not follicular B cells (Fig. 3D). In order to further subdivide B cells, we used CD23 co-staining to subdivide marginal zone and follicular B cells, and $\lg \mathrm{D}$ to subdivide follicular B cells. D-1MT decreased both transitional 1 (T1) and transitional 2 (T2) B cell subsets (Fig. 3E), with an increase in marginal zone B cells (Fig. 3F). 
Conversely, D-1MT did not impact splenic follicular or marginal zone precursor B cell numbers (Fig. 3F-G). Thus, D-1MT decreased the splenic transitional B cell pool, which suggested that D1MT impacted TIL-B cells via a systemic decrease of B cells.

\section{ID01 pathway inhibition leads to a partial block in B cell development}

To determine how D-1MT decreased B cells systemically, we investigated its effects on B cell development within the bone marrow in tumor bearing mice. There was a marked decrease

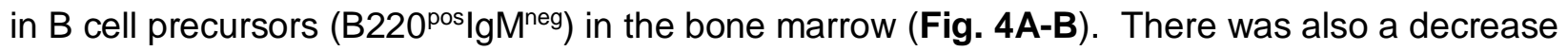
in pro-B and pre-B cells, together with an enrichment in the immature prepro-B cell compartment within the total B cell precursor pool (Fig. 4D-E, schematic of B cell development in Fig. 4C). Consistent with this, D-1MT increased the ratio of prepro-B cells relative to more mature $\mathrm{B}$ cell precursors (Fig. 4F). Together, these data demonstrate that treatment with D-1MT decreases pro-B and pre-B cells within the bone marrow, suggesting that D-1MT imposes a partial block in B cell development.

\section{D-1MT decreases intratumoral MDSCs, which regulate TIL-B cells}

To dissect the role of IDO1 on other immune cells in the tumor microenvironment that may modulate TIL-B cells, we investigated MDSCs. MDSCs modulate B cell responses ${ }^{58-60}$; conversely, B cells modify MDSC function ${ }^{61}$, and MDSCs express IDO1 in some settings ${ }^{62-64}$. Whether MDSCs are essential for TIL-B cell maintenance is unknown. Similar to B cells, we found that D-1MT decreases MDSCs (CD11 $\left.\mathrm{b}^{\text {pos }} \mathrm{Gr}-1^{\text {pos }}\right)$ in mice bearing B16F10 (Fig. 5A) and LLC tumors (Fig. 5B). Further, D-1MT decreases splenic MDSCs in B16F10 (Fig. 5C) and LLC tumors (Fig. 5D). Because prior studies showed that MDSCs influence B cell function ${ }^{59,65,66}$, and D-1MT decreased both TIL-B cells and MDSCs, we sought to determine whether MDSCs are required for TIL-B cell maintenance in our model. To this end, MDSCs were depleted in vivo, using an anti-Gr1 antibody (Fig. 5E). Consistent with the known function of anti-Gr1 antibody, 
this treatment led to a significant decrease in both intratumoral and splenic MDSCs (Sup. Fig. 4A-B). Strikingly, systemic MDSC depletion resulted in a decrease in TIL-B cells (Fig. 5F), suggesting that MDSCs are required for TIL-B cell maintenance. Conversely, anti-Gr1 antibody increased the percentage of splenic B cells (Fig. 5G), unlike IDO1 pathway inhibition which decreased splenic B cells in melanoma bearing mice (Fig. 3A). Thus, the IDO1 pathway maintains intratumoral and systemic MDSCs, and MDSCs drive intratumoral (but not systemic) B cell maintenance.

\section{D-1MT treatment and tumor Ido1 deletion have disparate immunologic impact}

As D-1MT mediated modest effects on tumor growth with concomitant decrease in TIL-B cells and MDSCs, we studied whether these effects were due to tumor IDO1 via implantation of Ido1 knockdown tumors. As Ido1 is an inducible enzyme, we confirmed Ido1 knockdown using interferon gamma stimulation in vitro (Sup. Fig. 5). Similar to B16F10 tumors in mice treated with $\mathrm{D}-1 \mathrm{MT}$, we found a modest (but significant) decrease in tumor size in Ido1 knockdown tumors (Fig. 6A). We confirmed Ido1 knockdown in vivo at time of tumor harvest (Fig. 6B). Unlike D-1MT treated B16F10 tumors, we found tumor specific Ido1 deficiency led to a significant increase in CD45+ tumor infiltrating leukocytes (Fig. 6C) and no difference in CD19+ TIL-B cells (Fig. 6D), MDSCs (Fig. 6E), or T cells (Fig. 6F). Thus, while D-1MT and knockdown of tumor Ido1 expression similarly impact tumor size, these interventions have disparate effects on B cells, T cells, and MDSCs.

\section{IDO1 is expressed by TIL-B cells}

To determine whether IDO1 expression is associated with B cells in human cancers, we interrogated human tumors from The Cancer Genome Atlas (TCGA) for IDO1 and B cell gene signature (BCGS) expression, using a validated BCGS ${ }^{67}$. We examined bladder cancer (BLCA), lung adenocarcinoma (LUAD), squamous cell carcinoma of the lung (LUSC), and melanoma 
(SKCM) and found each of these tumors segregated into two groups based on BCGS expression (low or high, Fig. 7A). Thus, we used the Partitioning Around Medoids (PAM) clustering algorithm to divide each tumor subtype into two groups based on high or low BCGS expression, and found intratumoral IDO1 expression was significantly greater in tumors with high BCGS expression (Fig. 7B). To determine whether IDO1 is expressed by human TIL-B cells, we interrogated a publicly available dataset where tumors from melanoma patients were analyzed by scRNAseq ${ }^{68}$. Of all TIL-B cells in analyzed patient tumors, 377 of 515 ( 73\%) expressed IDO1 (Fig. 7C, left and Fig. 7E). However, using another dataset ${ }^{69}$, we found minimal (13 of 1379 , or $0.94 \%$ ) TIL-B cells expressed IDO1 (Fig. 7C, right and Fig. 7E). To determine if this discrepancy was due to disparate sensitivity for IDO1 detection, we plotted IDO1 expression in all cells from analyzed tumors. While many cells (3246 of 4645 , or $\sim 70 \%$ ) express at least low levels of IDO1 in the Tirosh dataset (Fig. 7D, left and Fig. 7F), comparatively few cells (278 of 16291 , or $1.7 \%$ ) express IDO1 in the Sade-Feldman dataset (Fig. 7D, right and Fig. 7F), which primarily report cells that express high IDO1. The limited number of IDO1 positive cells in TIL-B cells from the SadeFeldman dataset may be due to technical limitations with detection of low expression of IDO1, which are robustly identified in the Tirosh dataset. Thus, TIL-B cells appear to express low levels of IDO1 in patients with melanoma.

\section{Discussion}

IDO1 is considered a critical regulator of effector T cell responses in humans and mice ${ }^{70-}$ 72. IDO1 regulates innate and adaptive immune responses in settings that include maternal tolerance to paternal allo-antigens in pregnancy, control of autoimmunity, mucosal tolerance, chronic infection states, and others ${ }^{70,73-75}$. In cancer, the role of IDO1 in suppressing T cell antitumor immunity was strengthened by preclinical murine models ${ }^{24,25,76}$ and early phase clinical trials demonstrating efficacy of IDO1 inhibitors in combination with other agents ${ }^{77,78}$. Our study 
demonstrates a novel role for IDO1 in regulating the B cell compartment in cancer, both systematically and within established tumors.

The modest impact of IDO1 inhibition or tumor Ido1 knockdown on tumor weight was expected, as the vast majority of preclinical and clinical studies support that IDO1-ablating treatments require combination with other therapies (chemotherapy, radiation, other immunotherapies) for significant anti-tumor effects $24,25,76,79$. Indeed, the best tumor response achieved in the phase I clinical trials using IDO1-inhibitor monotherapy was stable disease ${ }^{42,80}$.

The striking result in the current report is that IDO1 plays an important role in the regulation of systemic and intra-tumoral B cell subsets, and this effect may be mediated, at least in part, by MDSCs. We show for the first time that both D-1MT and linrodostat decrease intratumoral B cells. Recent studies have demonstrated that increased expression of a BCGS correlates with improved response to immune checkpoint inhibitor $(\mathrm{ICl})$ therapy in patients with advanced melanoma ${ }^{19}$. Moreover, B cells are essential for $\mathrm{ICI}$ treatment to slow tumor growth in a mouse model of triple negative breast cancer, and this therapy is approved for use in humans ${ }^{81,82}$. Our analysis of scRNAseq data in a preclinical melanoma model suggests that most TIL-B cells in untreated tumors are stimulatory. This further suggests that combining IDO1 inhibition with ICls may not be advantageous after all ${ }^{83}$.

Conversely, TIL-B cells are also associated with acquired resistance to BRAF-targeted therapy in melanoma ${ }^{50}$. Thus IDO1-inhibitor mediated TIL-B cell depletion may be beneficial in this setting, even as adjunctive therapy. Our data are also consistent with a report demonstrating IDO1 pathway inhibition attenuates pathology in a mouse model of rheumatoid arthritis, in part, by decreasing autoreactive $\mathrm{B}$ cells ${ }^{84}$. As metabolism of the amino acid glutamine is critical for $\mathrm{B}$ cell survival in certain conditions ${ }^{16}$, the role of IDO1 in regulating B cell development may be related to kynurenine depletion, and merits further study in rheumatologic conditions. 
Our results demonstrating a decrease in MDSCs following pharmacologic IDO1 pathway inhibition is consistent with previous reports ${ }^{62,85}$. However, there is a discordance between genetic and pharmacologic models with both MDSCs and B cells, as genetic deletion of tumor Ido1 does not impact tumor B cell or MDSC infiltration. Study of the IDO1-KO mouse in multiple settings, including pregnancy and implanted tumors, strongly suggest that these mice have developed compensatory mechanisms for the lack of IDO1 expression ${ }^{24,86}$. Consequently, robust reduction of tumor-infiltrating $\mathrm{B}$ cells and MDSCs via IDO1 inhibition may require IDO1 to first be upregulated in the tumor prior to IDO1-blockade. This is consistent with data that B cells and MDSCs can express IDO1 ${ }^{17,63}$, and tumor ldo1 over-expression increases TIL-MDSCs ${ }^{87}$.

Given that IDO1 appears to have a role in regulating MDSC tumor infiltration, it is remarkable that MDSCs themselves play a role in maintaining TIL-B cells. MDSCs can convert $B$ cells into Bregs ${ }^{60}$ and block $B$ cell proliferation and differentiation ${ }^{59,65,66}$. While this suggests that the absence of MDSCs could increase B cell numbers, we showed that MDSC depletion led to a decrease in TIL-B cells. Notably, MDSC depletion increased splenic B cells, suggesting MDSCs are not required to maintain systemic B cell populations. This is likely distinct from IDO1mediated regulation of MDSCs, as IDO1 pathway inhibition decreased both TIL-B and splenic B cells. How MDSCs regulate TIL-B cells is an area of active study and may result in additional therapeutic targets for clinical investigations.

\section{Acknowledgements}

The authors gratefully acknowledge support from the Conquer Cancer Foundation of the American Society of Clinical Oncology Young Investigator Award (to B.A.J.), the Harry J. Lloyd Charitable Trust Career Development Award (to B.A.J.), the AACR-BMS Fellowship for Young Investigators in Translational Immuno-oncology (to B.A.J.), the Alpha Omega Alpha Postgraduate Fellowship Award (to B.A.J.), two Johns Hopkins Physician Scientist Training Program Microgrant Awards (to B.A.J.), and the Johns Hopkins Institutional Training Grant in Medical Oncology (to 
B.A.J., NIH 5T32 CA009071, B.H. Park, PI). Initial studies were also supported by a NIH NRSA Predoctoral F30 award and an American Medical Association Foundation Seed Grant (both to B.A.J.). Further support was also provided by R01 CA235681 (to N.M.H.), and the Greenberg Bladder Cancer Institute. Dr. Johnson would also like to thank his patients, some of whom also graciously contributed financially to experiments published in this work.

\section{Conflict of Interest Statement}

No conflicts of interest to disclose.

\section{Dedication}

This paper is dedicated to Ross C. Donehower, MD, Professor, Director of Medical Oncology, and Director of the Medical Oncology / Hematology Fellowship Program, Johns Hopkins School of Medicine, with gratitude for his superior mentorship and impact on B.A.J.'s career development.

\section{Author Contributions}

B.A.J. conceived the study, designed, supervised, and executed experiments, analyzed data, and wrote the paper. K.A., J.M., W.Z., L.X., L.L., L.C., O.R., and D.W. performed experiments. D.D. and S.D. provided reagents/analytical tools. B.A.J., K.A., D.W., W.Z., L.L., L.X., L.C., O.R., D.D., S.D., T.S.J., N.H., D.J.M., and L.M.S.R. analyzed data. T.S.J. designed and performed pilot experiments. N.H., D.J.M., and T.S.J. revised the paper. L.M.S.R. supervised experiments, analyzed data, and wrote the paper. 

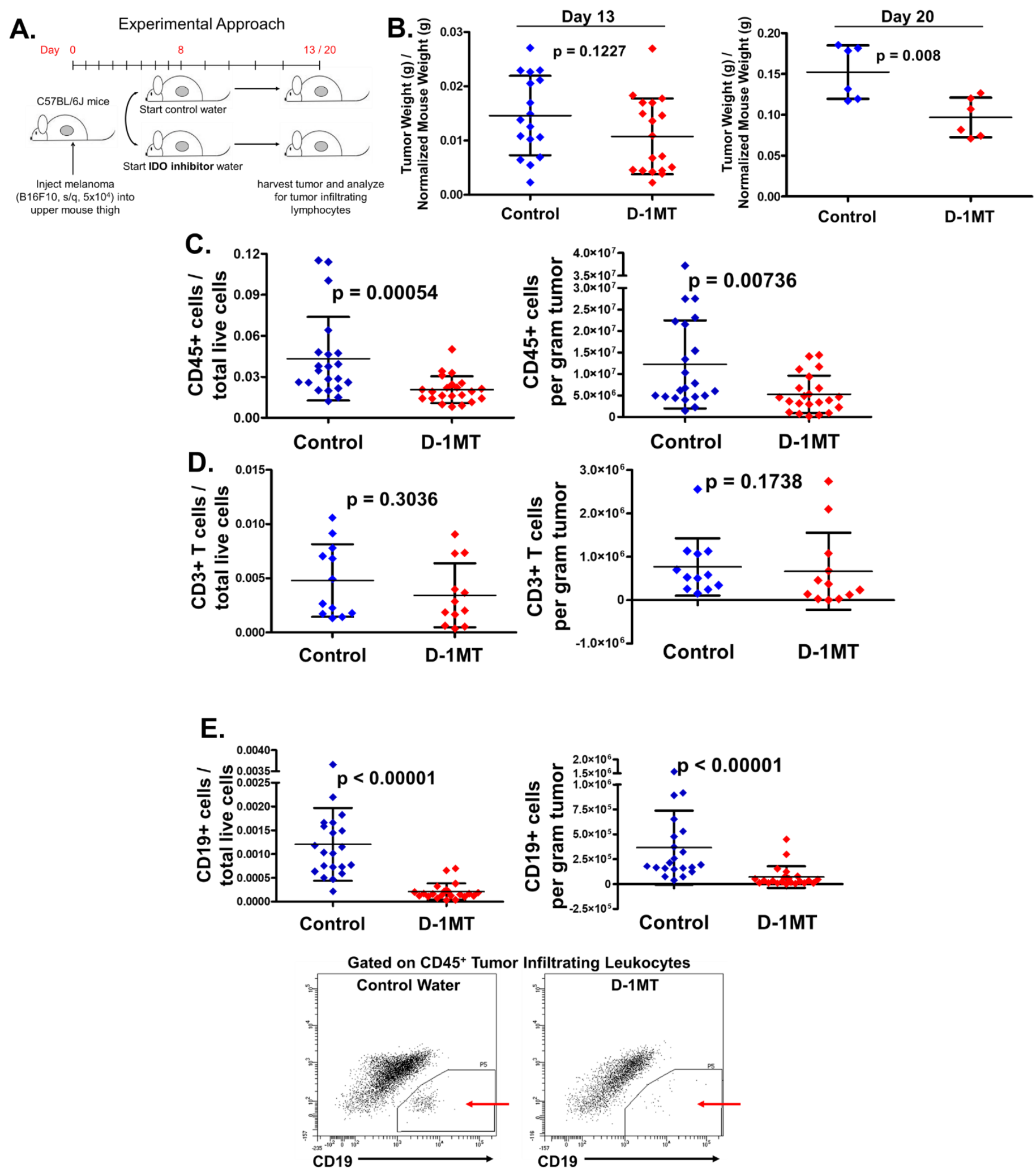

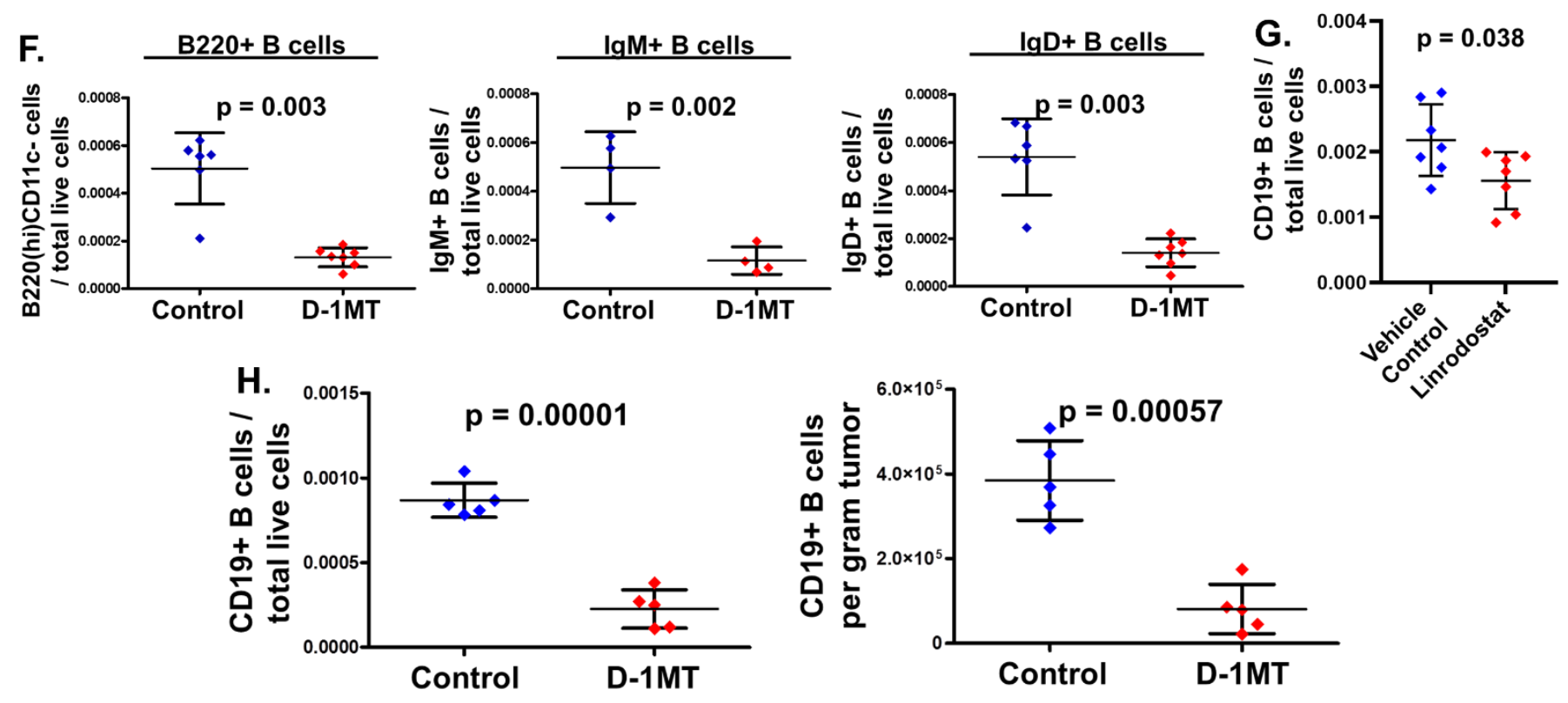

Gated on CD45+ Tumor Infiltrating Leukocytes
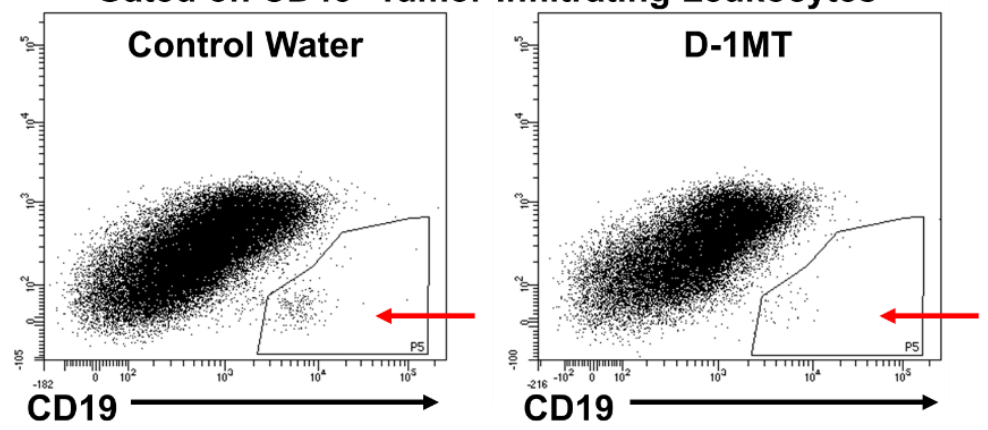

Figure 1. The ID01 pathway regulates TIL-B cells. A. Treatment schema. D-1MT or control water was started on day 8, and tumors were harvested for flow analysis on day 13. B. B16F10 tumor weights at day 13 and 20. For day 20 harvest, mice received control or D-1MT water for 5 continuous days followed by 2 days of standard water, in order to minimize previously described dehydration that occurs with $\mathrm{D}-1 \mathrm{MT}^{72}$ and similar to other protocols that give a 2 day break after 5 days of D-1MT treatment ${ }^{24}$. C-E. B16F10 tumors were harvested on

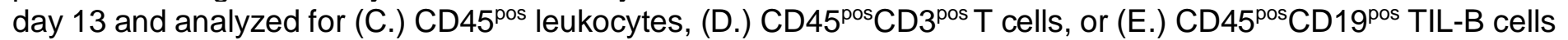
(with representative flow dot plots of TIL-B cells). F. Melanoma infiltrating CD45 ${ }^{\text {pos }}$ cells from day 13 tumors were analyzed for $\mathrm{B}_{22} 0^{\text {pos }} \mathrm{CD} 11 \mathrm{c}^{\text {neg }}$ (left), IgM ${ }^{\text {pos }}$ (center), and $\operatorname{lgD} \mathrm{D}^{\text {pos }}$ (right) B cells. G. B16F10 tumors were treated as in (A.) except on day 8 vehicle control or linrodostat was started daily via oral gavage. Day 13 tumors were analyzed for CD45 ${ }^{\text {pos }}$ CD19 $19^{\text {pos }}$ TIL-B cells. H. LLC tumors were injected and harvested as in (A.) and analyzed for $\mathrm{CD} 45^{\mathrm{pos}} \mathrm{CD} 19^{\text {pos }} \mathrm{B}$ cells. Each data point refers to a single mouse, and error bars represent \pm standard deviation. 

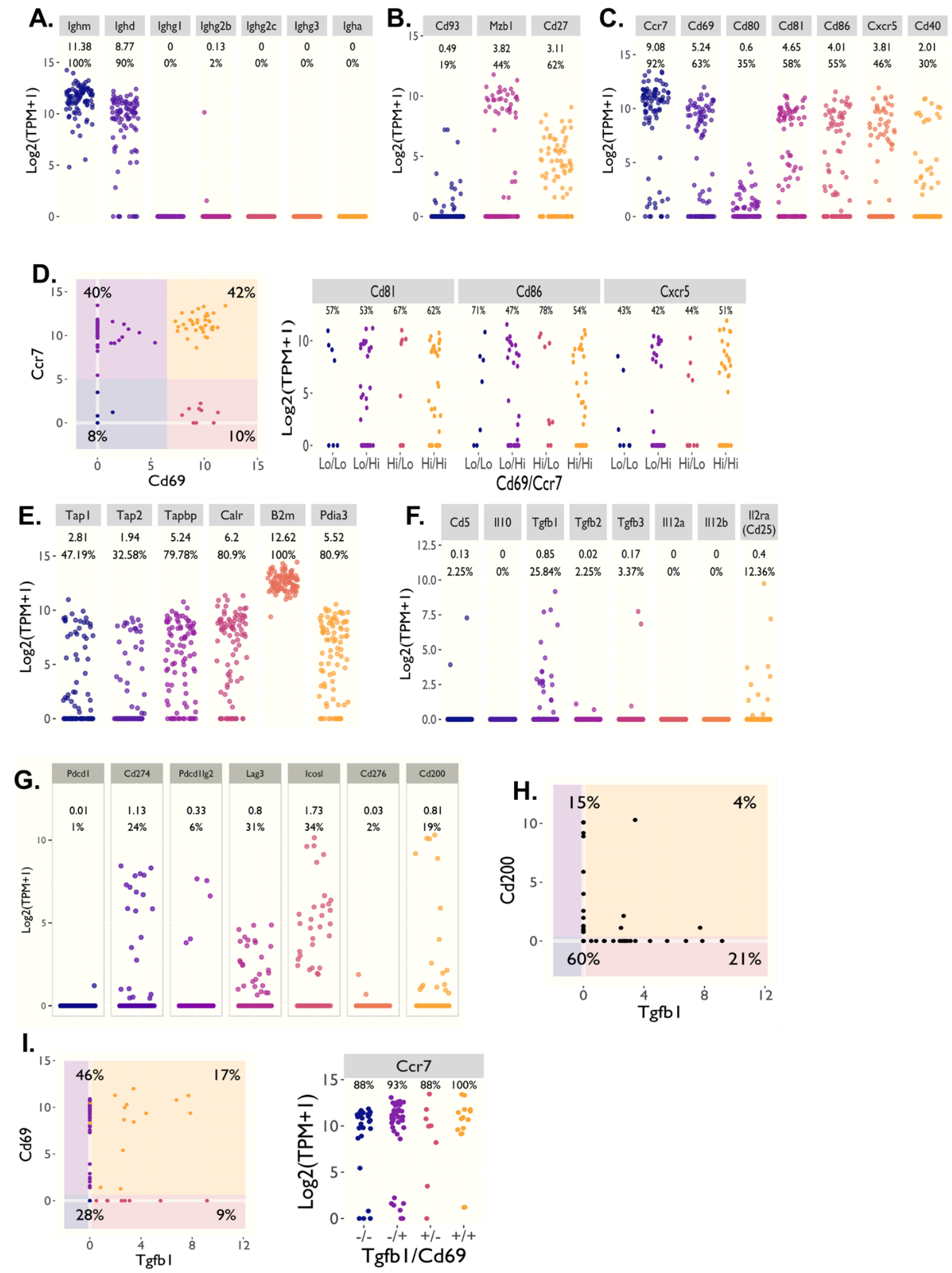
Figure 2. Melanoma TIL-B cells are phenotypically and functionally heterogeneous. ScRNAseq data from B16F10 tumors was accessed from a publicly available dataset ${ }^{31}$ and TIL-B cells analyzed for the following phenotypic and functional genes: A. Surface immunoglobulin genes, B. Cd93 (left), Mzb1 (center), and Cd27 (right). C. Genes associated with B cell activation. D. TIL-B cells were graphed based on expression of Ccr7 and Cd69 (left), and clusters based on Cd69 / Ccr7 expression were graphed based on Cd81 (left), Cd86 (center), and Cxcr5 expression (right). E-G. TIL-B cells were graphed based on expression of (E.) antigen processing genes, (F.) immune suppressive genes, and (G.) genes encoding checkpoint molecules. H. TIL-B cells were plotted based on expression of Tgfb1 and Cd200. I. TIL-B cells were plotted based on Tgfb1 and Cd69 expression, and clusters based on Tgfb1 / Cd69 expression colored based on Ccr7 expression. 

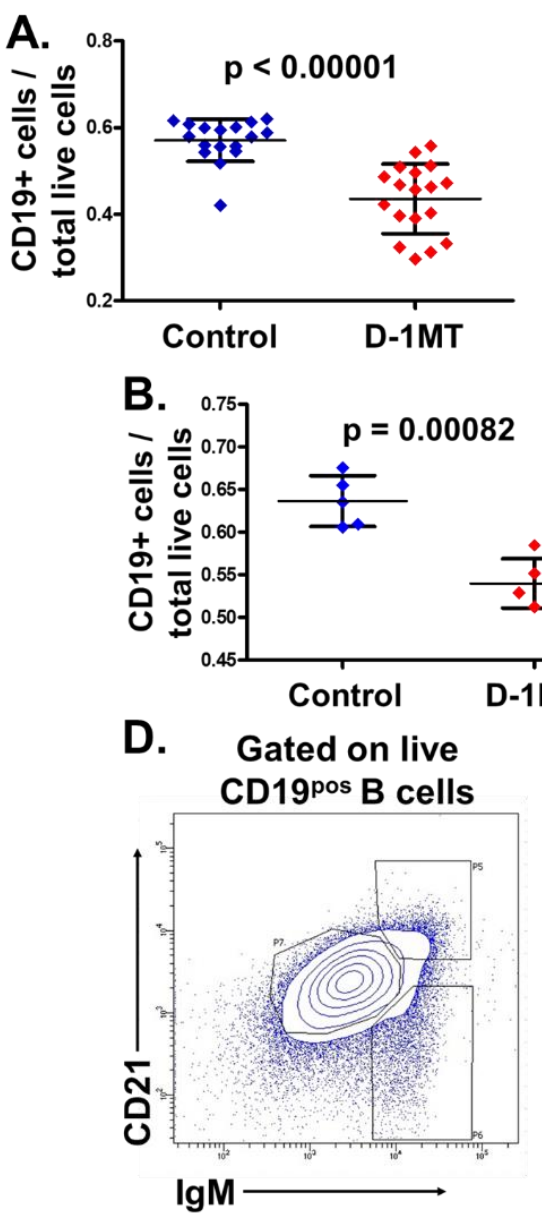
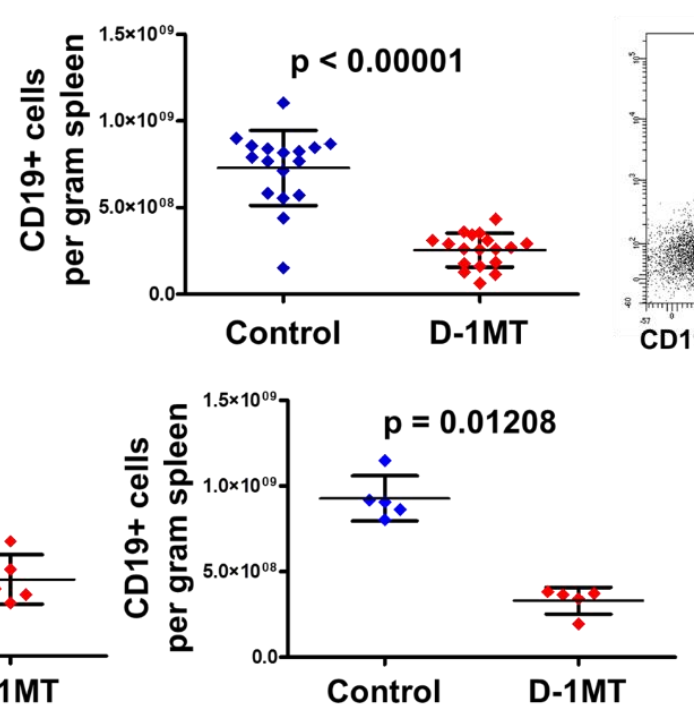

$$
\begin{aligned}
& \text { Ig } M^{\text {high }} C D 21^{\text {high }} \\
& \text { (MZ } \text { Ig }^{\text {high }} C D 21^{\text {low }}
\end{aligned}
$$

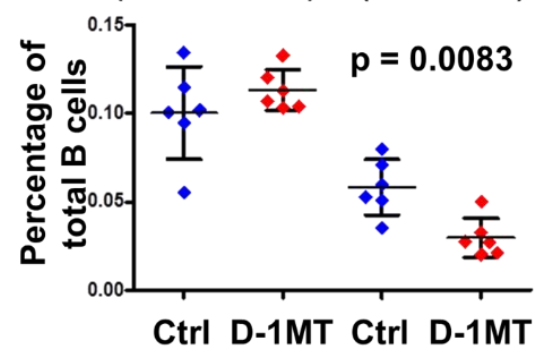

Gated on Live Splenocytes
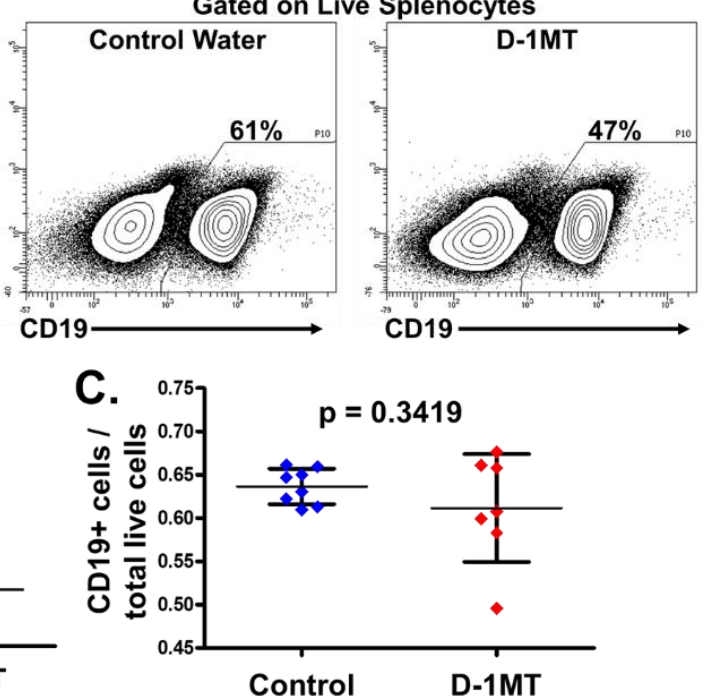

ntrol

$\lg M^{\text {pos }}$ CD21 pos

(Follicular)

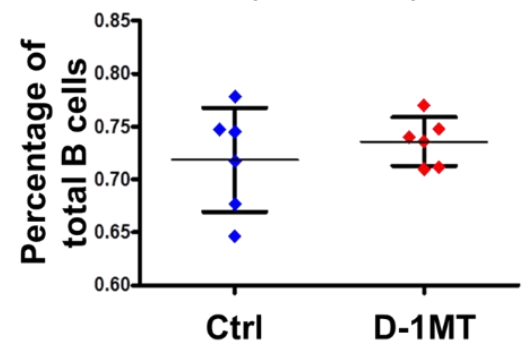

E. Ig $M^{\text {high }} C D 21^{\text {low }}$

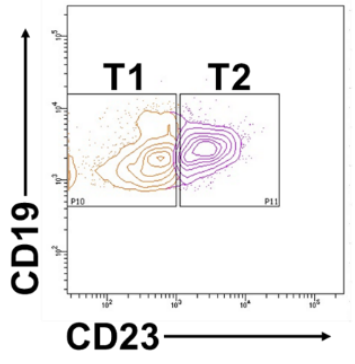

F. Ig $M^{\text {high }} C D 21^{\text {high }}$

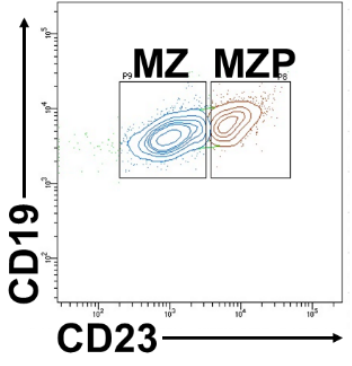

T1

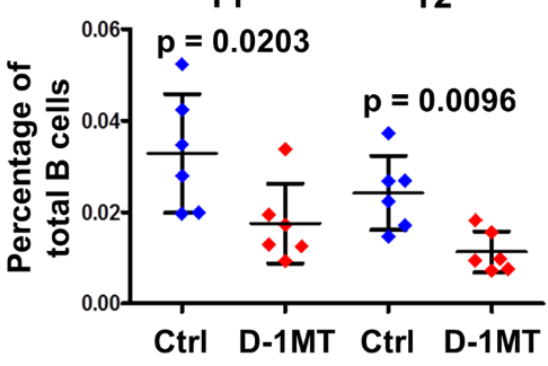

MZ

MZP

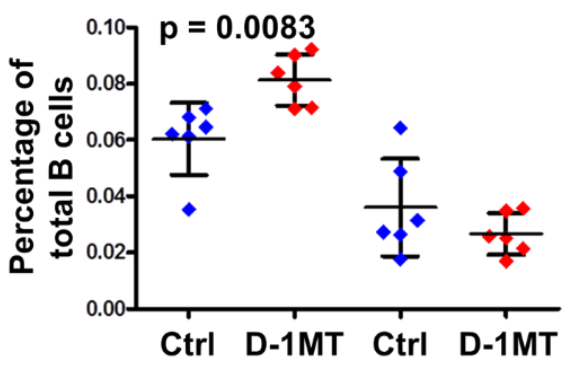



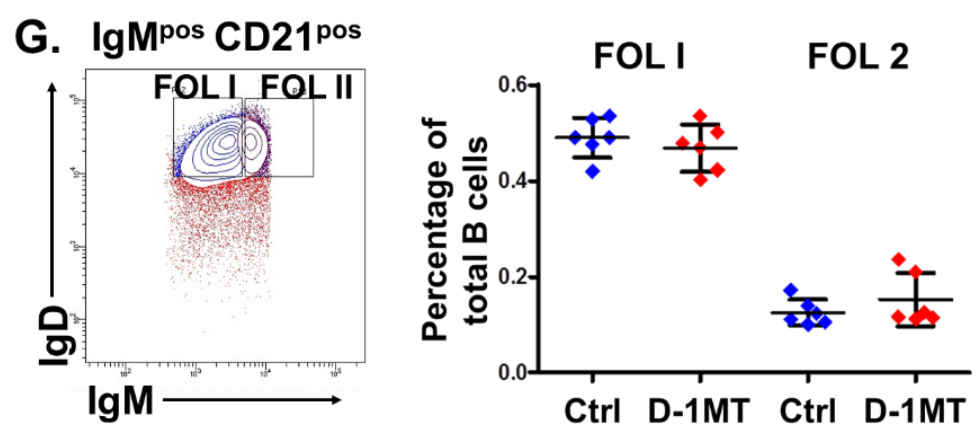

Figure 3. The IDO1 pathway regulates transitional B cells in melanoma bearing mice. Melanoma (B16F10) or Lewis Lung Carcinoma (LLC) cells were implanted into wild-type mice as in Fig. 1 (except Fig. 3C) and treated

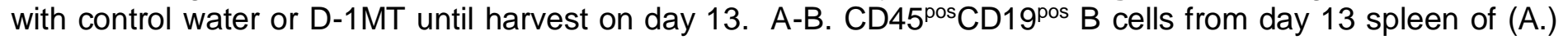
B16F10-bearing, (B.) LLC-bearing, or (C.) non-tumor-bearing mice were analyzed by flow cytometry. D. Splenic $B$ cells from B16F10-bearing mice were divided into lg $M^{\text {high }}$ CD21 ${ }^{\text {high }}$ (containing marginal zone $B$ cells, $M Z$ and marginal zone precursors, MZP), Ig ${ }^{\text {high }} C D 21^{\text {low }}$ (containing transitional B cells), and $\lg M^{\text {pos }} C D 21^{\text {pos }}$ (containing follicular B cells). E-G. Splenic B cells from the subdivisions described in (D.) were further divided into transitional (T)1 and T2 B cells (E.), MZ and MZP B cells (F.), and follicular (FOL) I and FOL II B cells (G.). 

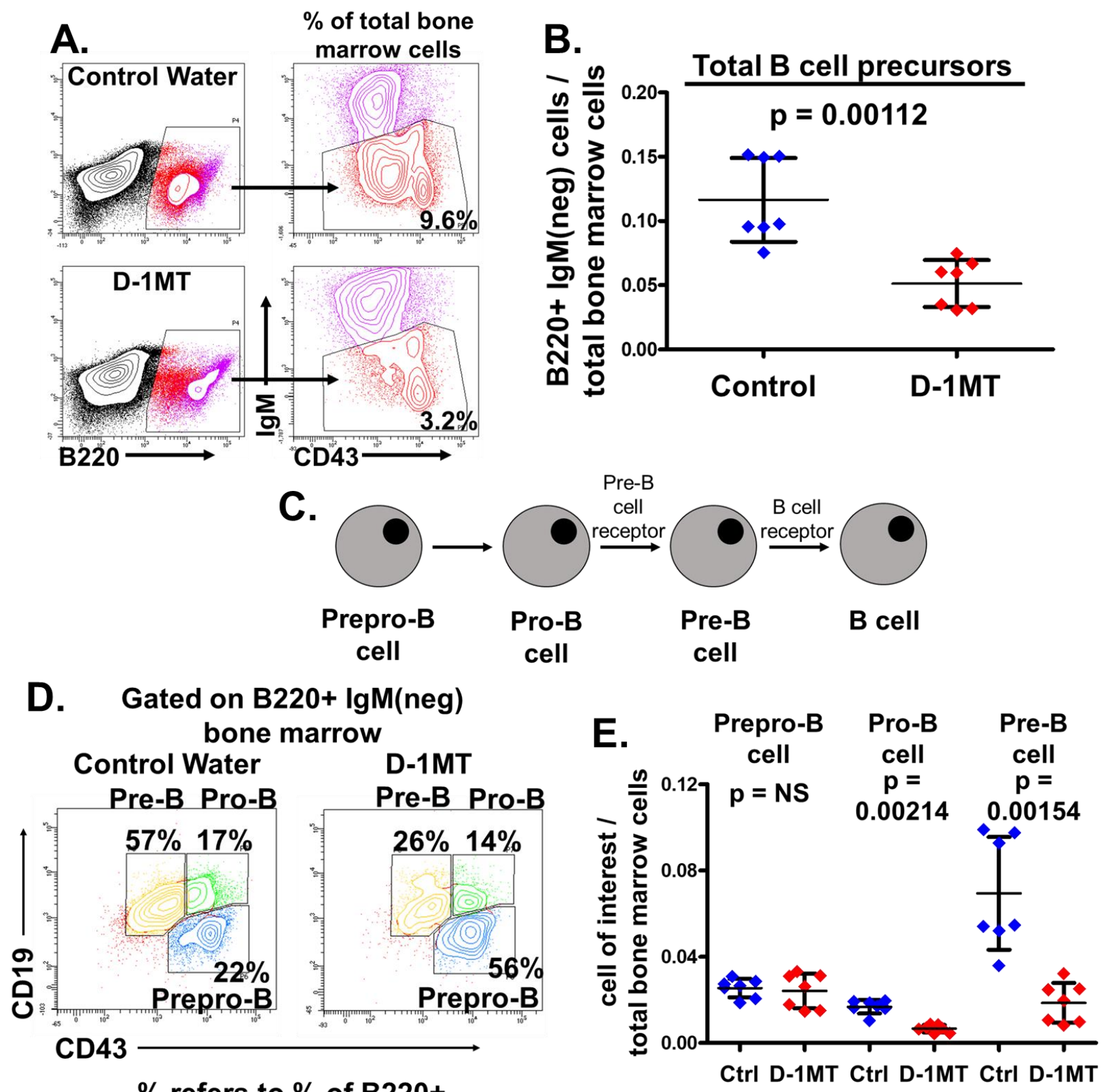

$\%$ refers to $\%$ of $\mathrm{B220+}$ IgM(neg) cells

D-1MT Pre-B Pro-B

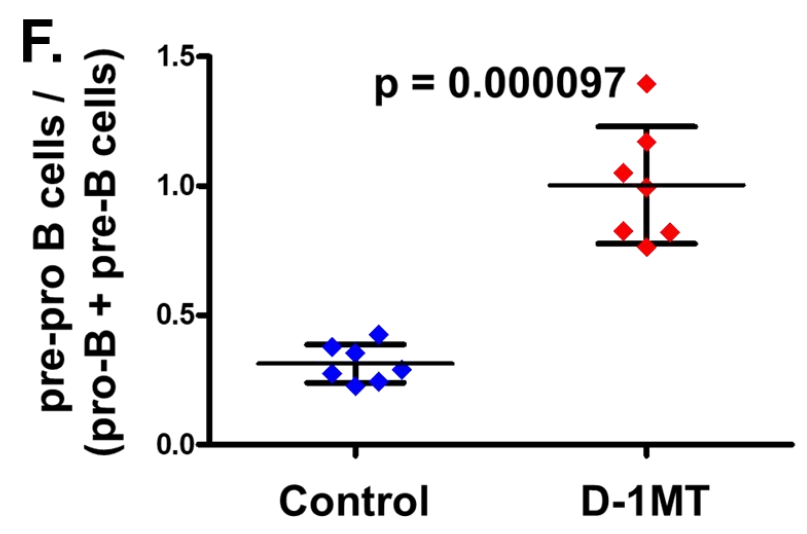


Figure 4. The IDO1 pathway regulates B cell precursors in melanoma-bearing mice. B16F10 tumors were implanted into wild-type mice as in Fig. 1A and treated with control water or D-1MT until harvest on day 13. A-B. Bone marrow from melanoma-bearing mice was harvested at day 13 and analyzed for $B 220^{\text {pos }} \operatorname{lgM}^{\text {neg }} \mathrm{B}$ cell precursors. C. Schematic of B cell development. D. B220 ${ }^{\text {pos }} \mathrm{gg}^{\text {neg }} \mathrm{B}$ cell precursors were analyzed for prepro$B$, pro-B, and pre-B subsets as shown. E. Total bone marrow cells were analyzed for prepro-B, pro- $B$, and preB cells. F. Ratio of pre-pro B cells to downstream pro-B and pre-B cells. Bars represent standard deviation. 

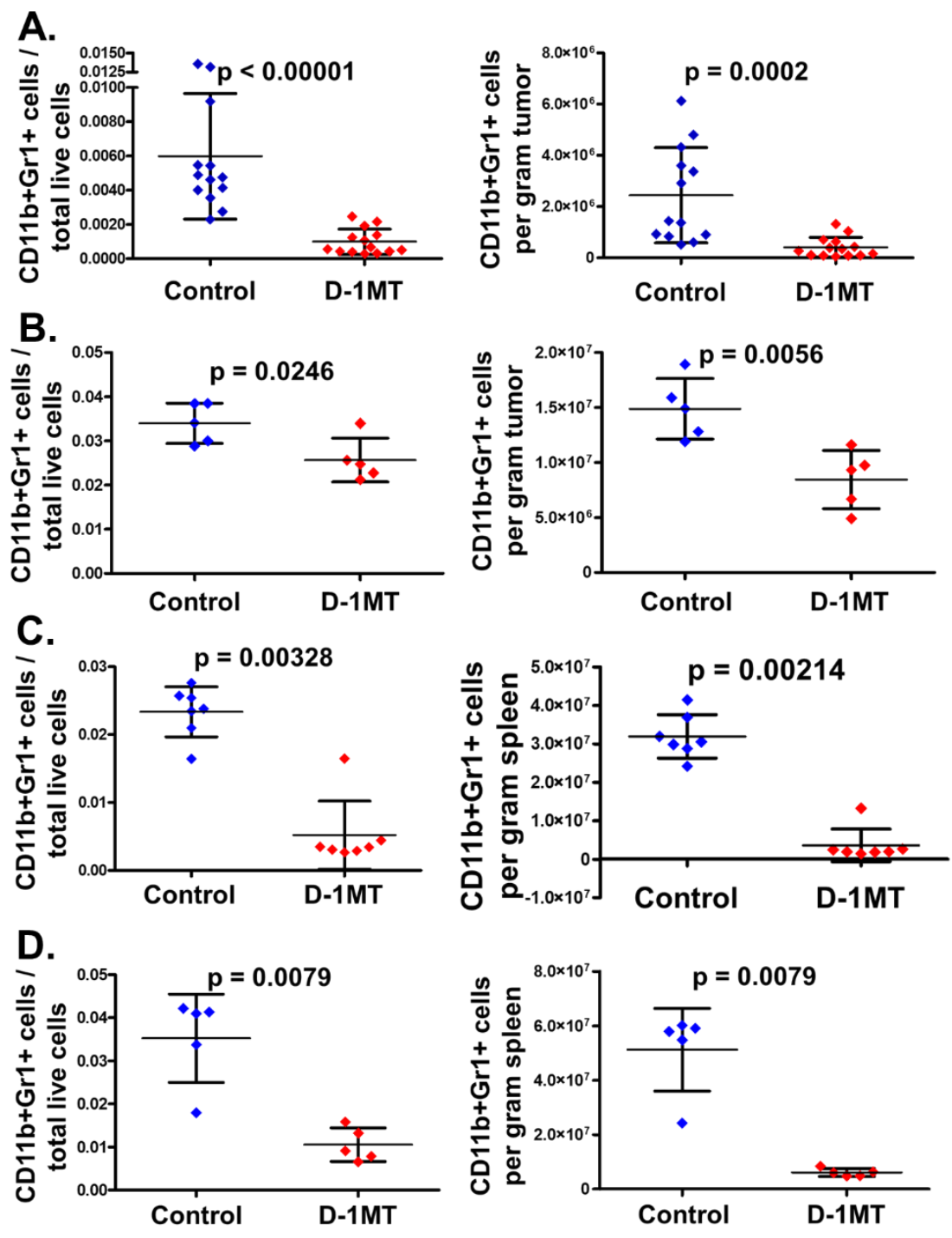

E.
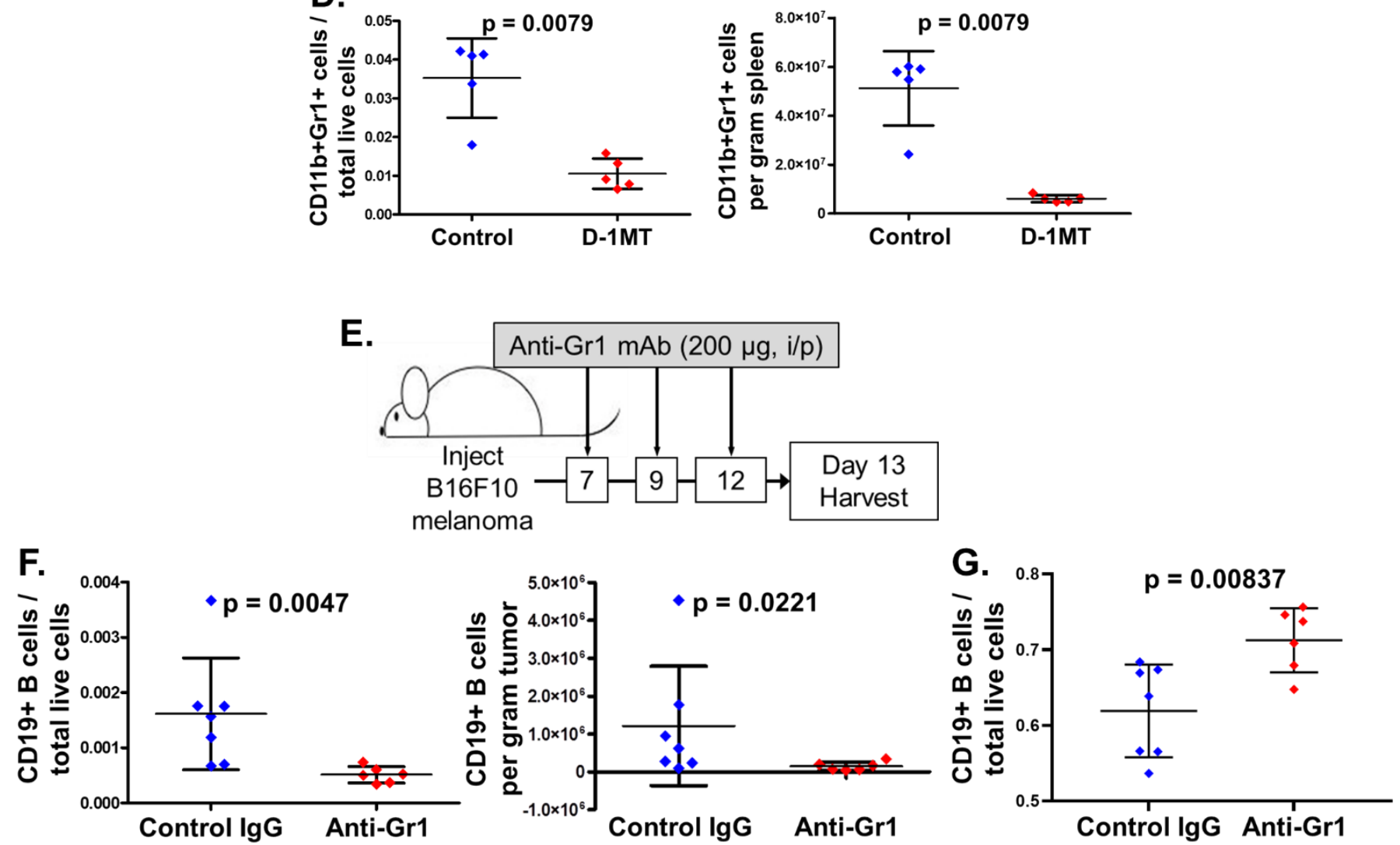
Figure 5. The ID01 pathway regulates MDSCs, which regulate TIL-B cell maintenance. Mice were injected with B16F10 or LLC as in Fig. 1, and on day 13 tumors from B16F10 (A.) and LLC (B.) were analyzed for CD45 ${ }^{\text {pos }} \mathrm{CD} 11 \mathrm{~b}^{\text {pos }} \mathrm{Gr}-1^{\text {pos }}$ MDSCs. C-D. Spleens from B16F10 (C.) and LLC (D.) tumor-bearing mice were analyzed for MDSCs. E. Treatment schema for MDSC depletion. Control lg or Anti-Gr1 antibody $(200 \mu \mathrm{g})$ was

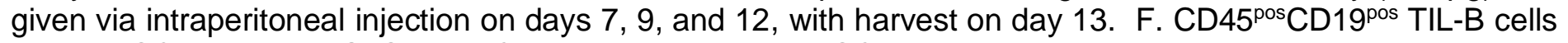
after MDSC depletion. G. Splenic CD19 ${ }^{\text {pos }}$ B cells after MDSC depletion. Bars represent standard deviation. 

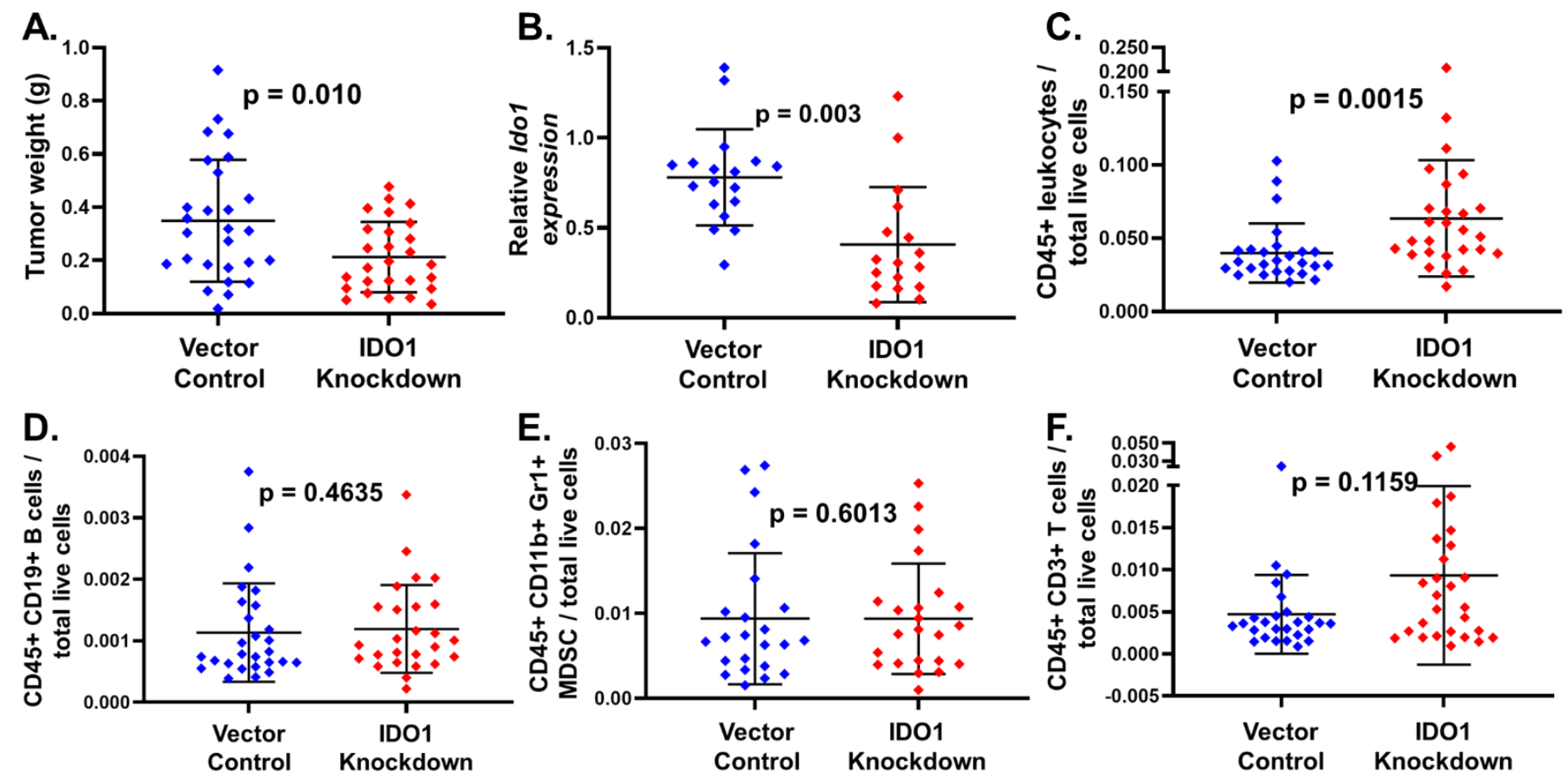

Figure 6. Ido1 tumor knockdown impacts the tumor microenvironment differently than IDO1 pathway inhibition. Vector control or $/ d o 1$ knockdown tumors were injected on day 0 and harvested on day 13. A. Tumor weights of vector control and Ido 1 knockdown tumors. B. Ido1 expression of vector control and Ido 1 knockdown tumors in vivo following tumor harvest on day 13. C-F. Day 13 tumors were analyzed for (C.) CD45+ leukocytes, (D.) CD45+CD19+ B cells, (E.) CD45+CD11b+Gr1+ MDSCs, and (F.) CD45+CD11b+CD3+ T cells. 

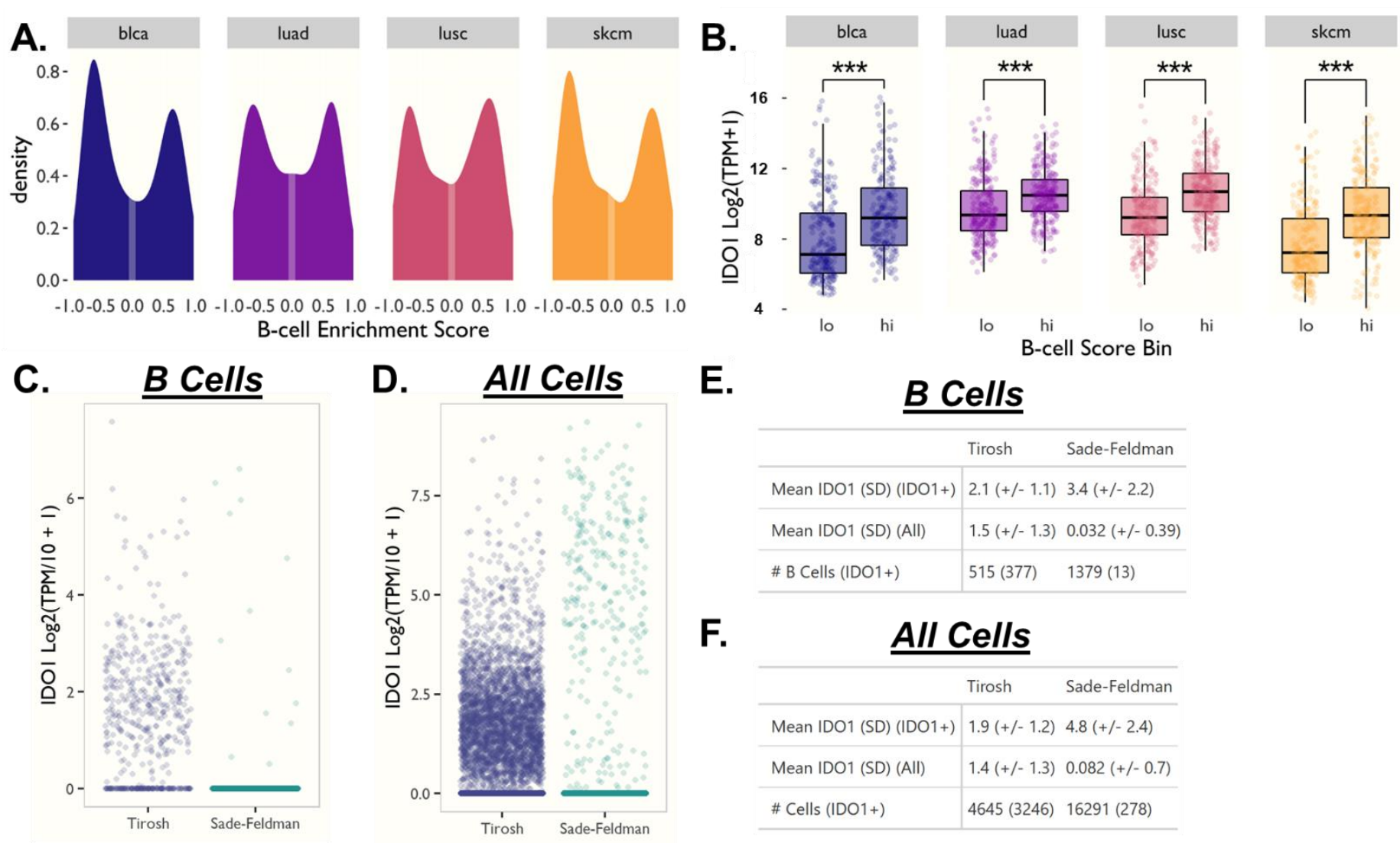

Figure 7. B cell gene signature (BCGS) expression correlates with IDO1 in human cancers, and human intratumoral B cells express IDO1. A. Bulk RNA sequencing data from tumors from TCGA muscle invasive bladder cancer (blca), lung adenocarcinoma (luad), lung squamous cell carcinoma (lusc), and melanoma (skcm) were analyzed and graphed based on expression of a validated BCGS (Danaher et al. ${ }^{67}$ ). B. Tumors from (A.) were examined for correlation of IDO1 with low and high levels of BCGS expression. ${ }^{* *}=p<0.001$. C-D. Single cell RNA sequencing of melanoma tumors from Tirosh (left) and Sade-Feldman (right) datasets were analyzed for IDO1 expression (C. B cells, D. all cells). E-F. Results from C-D. are presented in table format. 
A.
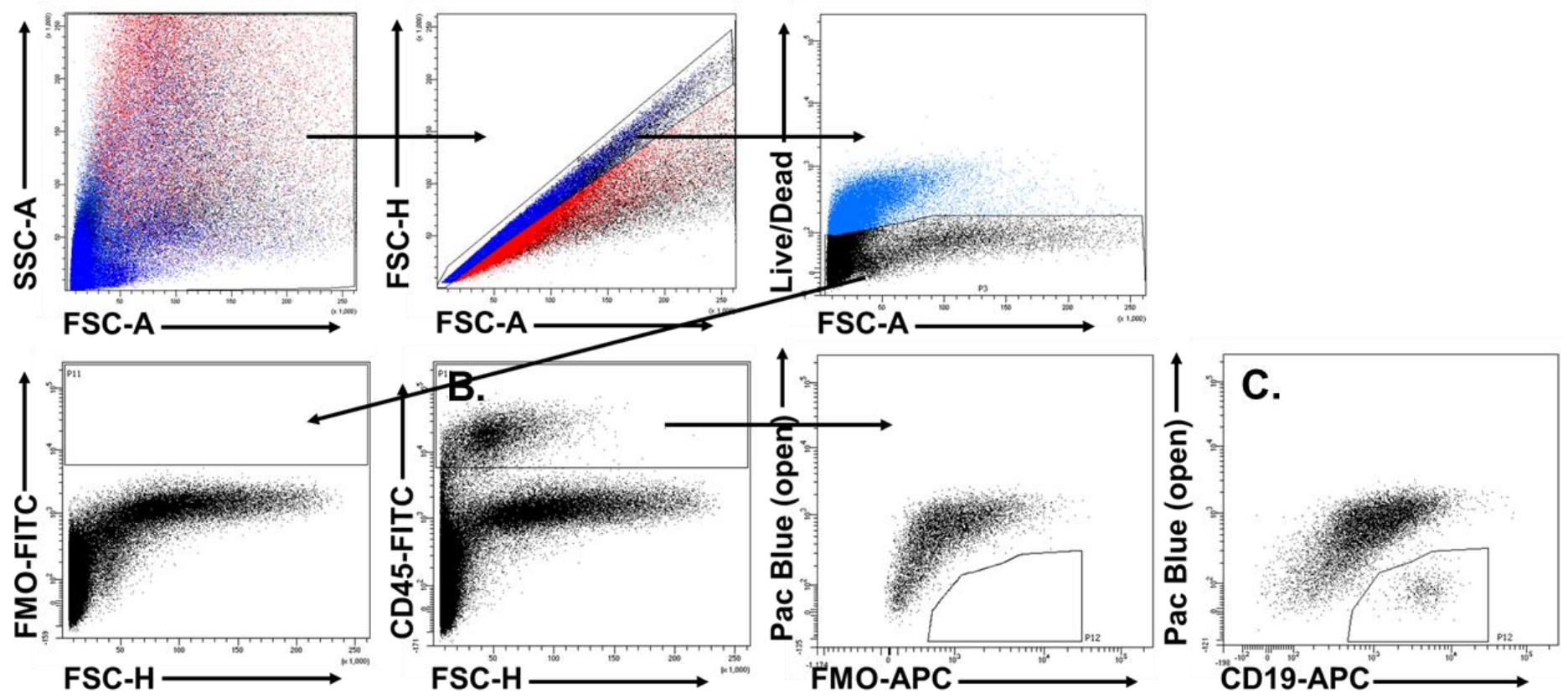

Supplemental Figure 1. Gating schema for intratumoral flow cytometry analysis. A. Doublets and dead cells were removed from analysis, and unstained controls were consistently used to set analysis gates (bottom left panel). B. Gating structure for CD45 ${ }^{\text {pos }}$ leukocyte analysis (left), which was used to set fluorescence minus one (FMO) control for CD19 staining (right). C. CD19 ${ }^{\text {pos }}$ staining after initially gating on CD45 ${ }^{\text {pos }}$ leukocytes. 

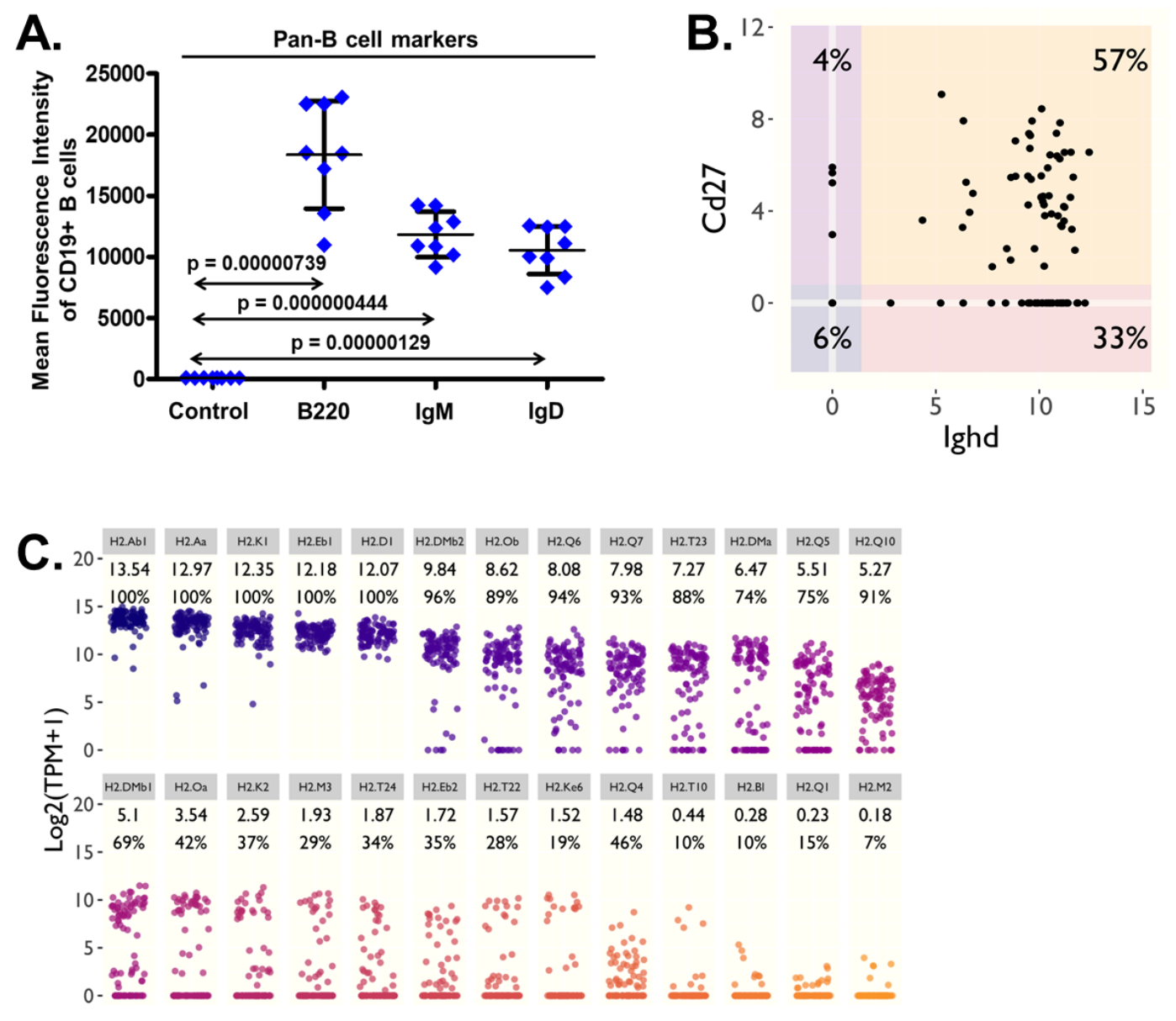

Supplemental Figure 2. A. CD45 ${ }^{\text {pos }}$ CD19pos TIL-B cells from B16F10 bearing mice were analyzed on day 13 for B cell markers. B-C. TIL-B cells were analyzed by scRNAseq data as in Fig. 2, except for (B.) Ighd and Cd27 (C.) the presence of $\mathrm{MHC}$ genes. 

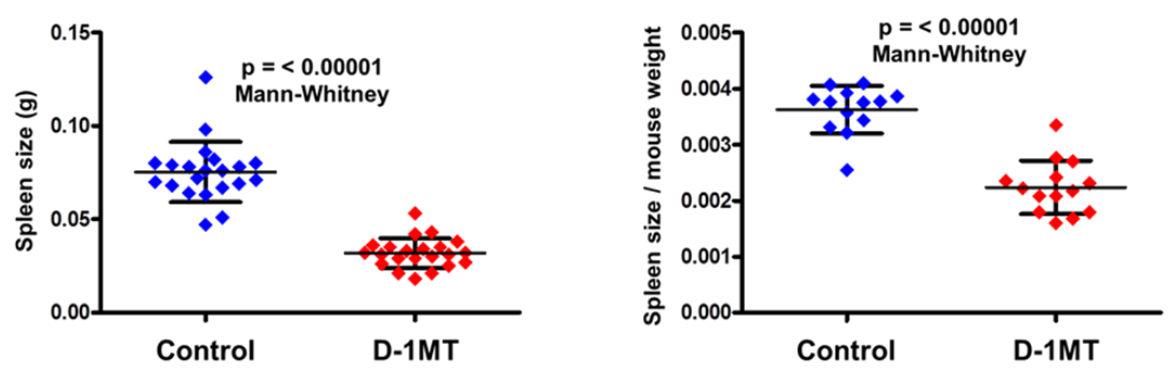

Supplemental Figure 3. Melanoma bearing mice were treated as in Fig. 1A. On day 13 spleens were weighed (left) and normalized to total mouse weight (right). 

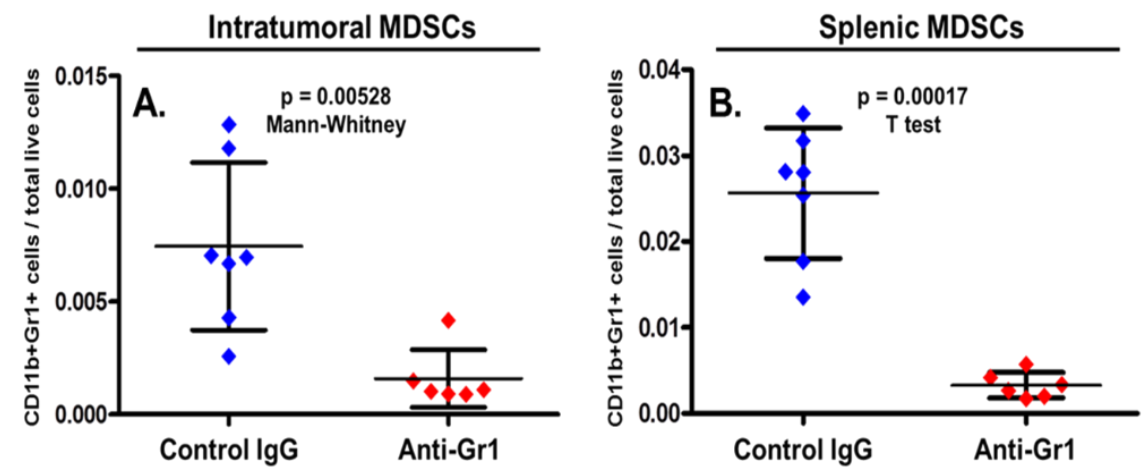

Supplemental Figure 4. Melanoma-bearing mice were treated as in Fig. 6C. On day 13 tumors (A.) and spleens (B.) were analyzed for CD11 $\mathrm{b}^{\text {pos }} \mathrm{Gr} 1^{\text {pos }}$ MDSCs.
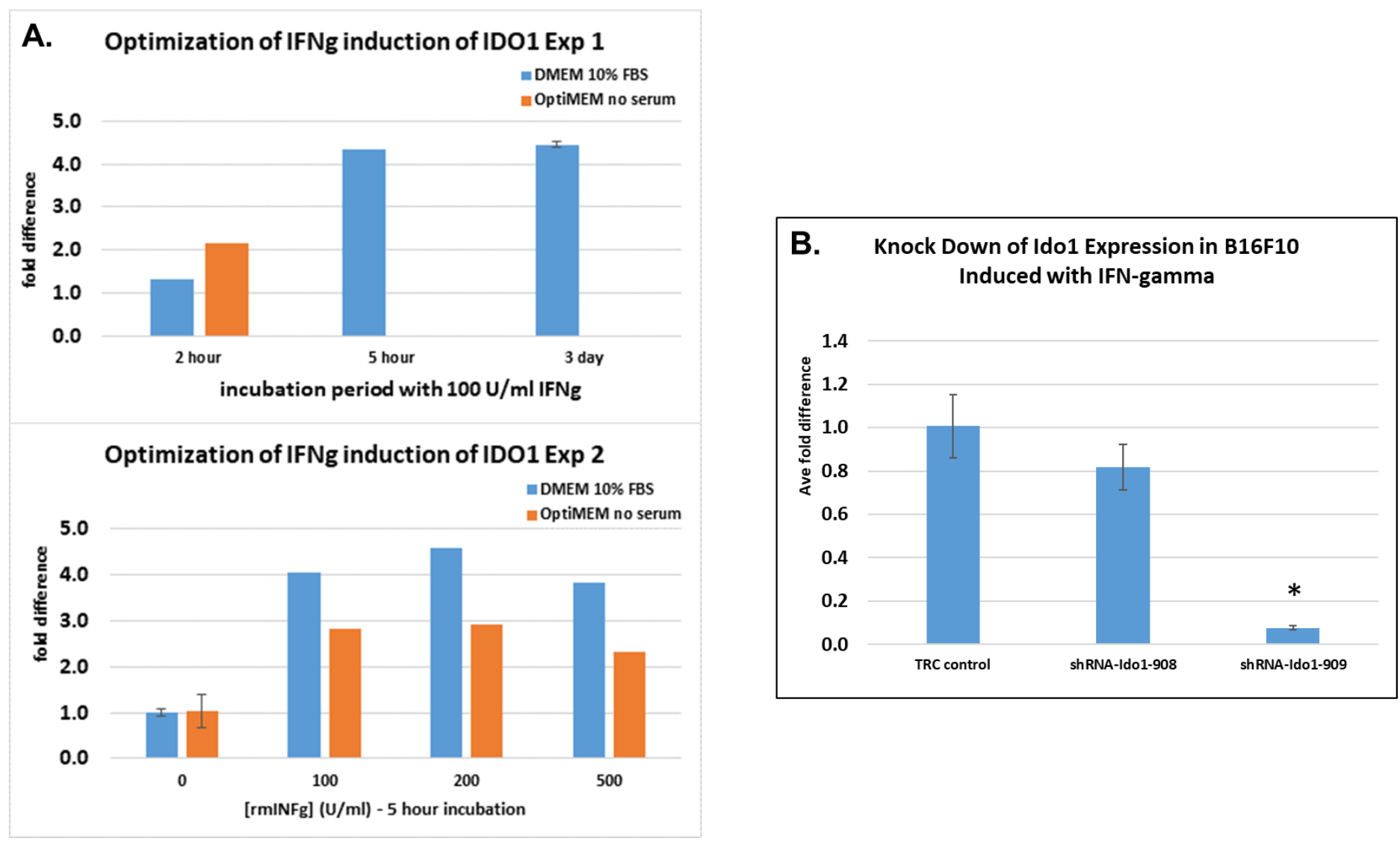

Supplemental Figure 5. A, top. B16F10 melanoma cells were treated in vitro with 100 units $/ \mathrm{mL}$ interferon gamma for 5 hours or 3 days then harvested and analyzed for Ido1 expression by PCR. A, bottom. B16F10 melanoma cells were incubated with 100,200 , or 500 units $/ \mathrm{mL}$ interferon gamma in media with or without serum as shown. After 5 hours tumor cells were harvested and analyzed for Ido1 expression by PCR. B. Ido1 expression levels of vector control and two different ldo1 knockdown cells following induction with interferon gamma for 5 hours. The 909 ldo1 knockdown was used for all in vivo experiments. 


\section{References:}

1. Dutta, S., Sengupta, P. \& Haque, N. Reproductive immunomodulatory functions of B cells in pregnancy. International Reviews of Immunology 39, 53-66 (2020).

2. Nothelfer, K., Sansonetti, P. J. \& Phalipon, A. Pathogen manipulation of B cells: The best defence is a good offence. Nature Reviews Microbiology 13, 173-184 (2015).

3. Rawlings, D. J., Metzler, G., Wray-Dutra, M. \& Jackson, S. W. Altered B cell signalling in autoimmunity. Nature Reviews Immunology 17, 421-436 (2017).

4. Sharonov, G. V., Serebrovskaya, E. O., Yuzhakova, D. V., Britanova, O. V. \& Chudakov, D. M. B cells, plasma cells and antibody repertoires in the tumour microenvironment. Nature Reviews Immunology 20, 294-307 (2020).

5. Rossetti, R. A. M. et al. B lymphocytes can be activated to act as antigen presenting cells to promote antitumor responses. PLoS One 13, e0199034 (2018).

6. Mauri, C. \& Menon, M. Human regulatory B cells in health and disease: therapeutic potential. J. Clin. Invest. 127, 772-779 (2017).

7. Yang, C. et al. B Cells Promote Tumor Progression via STAT3 Regulated-Angiogenesis. PLoS One 8, e64159 (2013).

8. Zhang, Y., Gallastegui, N. \& Rosenblatt, J. D. Regulatory B cells in anti-tumor immunity. Int. Immunol. 27, 521-30 (2015).

9. Zhou, X., Su, Y.-X., Lao, X.-M., Liang, Y.-J. \& Liao, G.-Q. CD19(+)IL-10(+) regulatory B cells affect survival of tongue squamous cell carcinoma patients and induce resting CD4(+) T cells to CD4(+)Foxp3(+) regulatory T cells. Oral Oncol. 53, 27-35 (2016).

10. Xiao, X. et al. PD-1hi Identifies a Novel Regulatory B-cell Population in Human Hepatoma That Promotes Disease Progression. Cancer Discov. 6, 546-559 (2016).

11. Qian, L. et al. Clinical significance of regulatory B cells in the peripheral blood of patients with oesophageal cancer. Cent. J. Immunol. 40, 263-5 (2015).

12. Liu, J. et al. Aberrant frequency of IL-10-producing B cells and its association with Treg and MDSC cells in Non Small Cell Lung Carcinoma patients. Hum. Immunol. 77, 84-9 (2016).

13. Li, W. et al. Reduction in Peripheral CD19+CD24hCD27+ B Cell Frequency Predicts Favourable Clinical Course in XELOX-Treated Patients with Advanced Gastric Cancer. Cell. Physiol. Biochem. 41, 2045-2052 (2017).

14. Boothby, M. \& Rickert, R. C. Metabolic Regulation of the Immune Humoral Response. (2017). doi:10.1016/j.immuni.2017.04.009

15. Kedia-Mehta, N. \& Finlay, D. K. Competition for nutrients and its role in controlling immune responses. Nature Communications 10, 1-8 (2019).

16. Le, A. et al. Glucose-independent glutamine metabolism via TCA cycling for proliferation and survival in b cells. Cell Metab. 15, 110-121 (2012).

17. Shinde, R. et al. B Cell-Intrinsic IDO1 Regulates Humoral Immunity to T CellIndependent Antigens. J. Immunol. 195, 2374-2382 (2015).

18. El-Zaatari, M. et al. Indoleamine 2,3-Dioxygenase 1, Increased in Human Gastric PreNeoplasia, Promotes Inflammation and Metaplasia in Mice and Is Associated With Type II Hypersensitivity/Autoimmunity. Gastroenterology 154, 140-153.e17 (2018).

19. Helmink, B. A. et al. B cells and tertiary lymphoid structures promote immunotherapy response. Nature (2020). doi:10.1038/s41586-019-1922-8

20. Munn, D. H. \& Mellor, A. L. Indoleamine 2,3-dioxygenase and tumor-induced tolerance. J. Clin. Invest. 117, 1147-54 (2007).

21. Lemos, H. et al. STING Promotes the Growth of Tumors Characterized by Low Antigenicity via IDO Activation. Cancer Res. 76, 2076-2081 (2016).

22. Berrong, Z. et al. Antigen-Specific Antitumor Responses Induced by OX40 Agonist Are Enhanced by the IDO Inhibitor Indoximod. Cancer Immunol. Res. 6, 201-208 (2018). 
23. Munn, D. H. et al. Expression of indoleamine 2,3-dioxygenase by plasmacytoid dendritic cells in tumor-draining lymph nodes. J. Clin. Invest. 114, 280-90 (2004).

24. Hou, D.-Y. et al. Inhibition of indoleamine 2,3-dioxygenase in dendritic cells by stereoisomers of 1-methyl-tryptophan correlates with antitumor responses. Cancer Res. 67, 792-801 (2007).

25. Holmgaard, R. B., Zamarin, D., Munn, D. H., Wolchok, J. D. \& Allison, J. P. Indoleamine 2,3-dioxygenase is a critical resistance mechanism in antitumor T cell immunotherapy targeting CTLA-4. J. Exp. Med. 210, 1389-402 (2013).

26. Kumar, V. et al. Cancer-Associated Fibroblasts Neutralize the Anti-tumor Effect of CSF1 Receptor Blockade by Inducing PMN-MDSC Infiltration of Tumors. Cancer Cell 32, 654668.e5 (2017).

27. Sharma, M. D. et al. The PTEN pathway in Tregs is a critical driver of the suppressive tumor microenvironment. Sci. Adv. 1, e1500845 (2015).

28. Herzenberg, L. A., Tung, J., Moore, W. A., Herzenberg, L. A. \& Parks, D. R. Interpreting flow cytometry data: a guide for the perplexed. Nat. Immunol. 7, 681-5 (2006).

29. Perfetto, S. P., Chattopadhyay, P. K. \& Roederer, M. Seventeen-colour flow cytometry: unravelling the immune system. Nat. Rev. Immunol. 4, 648-55 (2004).

30. Morgan, M. \& Davis, S. GenomicDataCommons: NIH / NCI Genomic Data Commons Access. (2020). Available at: https://bioconductor.org/packages/GenomicDataCommons.

31. Davidson, S. et al. Single-Cell RNA Sequencing Reveals a Dynamic Stromal Niche That Supports Tumor Growth. Cell Rep. 31, (2020).

32. Love, M. I., Huber, W. \& Anders, S. Moderated estimation of fold change and dispersion for RNA-seq data with DESeq2. Genome Biol. 15, (2014).

33. Hänzelmann, S., Castelo, R. \& Guinney, J. GSVA: Gene set variation analysis for microarray and RNA-Seq data. BMC Bioinformatics 14, (2013).

34. R. Core Team. R: A language and environment for statistical computing. (2020).

35. MacKenzie, C. J. \& Shioda, T. COS-1 cells as packaging host for production of lentiviruses. Curr. Protoc. Cell Biol. Chapter 26, (2011).

36. Benskey, M. \& Manfredsson, F. Lentivirus production and purification. in Gene Therapy for Neurological Disorders 107-114 (Humana Press, 2016).

37. Livak, K. J. \& Schmittgen, T. D. Analysis of relative gene expression data using real-time quantitative PCR and the 2- $\triangle \triangle C T$ method. Methods 25, 402-408 (2001).

38. Ryan, T. \& Joiner, B. Normal probability plots and tests for normality. (Pennsylvania State University, 1973).

39. D'Agostino, R. \& Pearson, E. Tests for departure from normality. Empirical results for the distributions of b2 and $\sqrt{ } b 1$. Biometrika 60, 613-622 (1973).

40. Snedecor, G. \& Cochran, W. Statistical Methods. (Iowa State University Press, 1976).

41. Jia, L. et al. Toxicology and pharmacokinetics of 1-methyl-d-tryptophan: Absence of toxicity due to saturating absorption. Food Chem. Toxicol. 46, 203-211 (2008).

42. Soliman, H. H. et al. A phase I study of indoximod in patients with advanced malignancies. Oncotarget 7, 22928-38 (2016).

43. Fox, E. et al. Indoximod: An Immunometabolic Adjuvant That Empowers T Cell Activity in Cancer. Front. Oncol. 8, 370 (2018).

44. Liu, X. et al. Selective inhibition of IDO1 effectively regulates mediators of antitumor immunity. Blood 115, 3520-30 (2010).

45. Prendergast, G. C., Malachowski, W. P., DuHadaway, J. B. \& Muller, A. J. Discovery of IDO1 Inhibitors: From Bench to Bedside. Cancer Res. 77, 6795-6811 (2017).

46. Metz, R. et al. IDO inhibits a tryptophan sufficiency signal that stimulates mTOR: A novel IDO effector pathway targeted by D-1-methyl-tryptophan. Oncoimmunology 1, 1460-1468 (2012).

47. Sharma, M. D. et al. Indoleamine 2,3-dioxygenase controls conversion of Foxp3+ Tregs 
to TH17-like cells in tumor-draining lymph nodes. Blood 113, 6102-11 (2009).

48. Shinde, R. et al. B Cell-Intrinsic IDO1 Regulates Humoral Immunity to T Cell-Independent Antigens. J. Immunol. 195, 2374-82 (2015).

49. Cabrita, R. et al. Tertiary lymphoid structures improve immunotherapy and survival in melanoma. Nature 577, 561-565 (2020).

50. Somasundaram, R. et al. Tumor-associated B-cells induce tumor heterogeneity and therapy resistance. Nat. Commun. 8, 607 (2017).

51. Allman, D. \& Pillai, S. Peripheral B cell subsets. Curr. Opin. Immunol. 20, 149-157 (2008).

52. De Guinoa, J. S., Barrio, L., Mellado, M. \& Carrasco, Y. R. CXCL13/CXCR5 signaling enhances BCR-triggered B-cell activation by shaping cell dynamics. Blood 118, 15601569 (2011).

53. Yanaba, K. et al. A regulatory B cell subset with a unique CD1dhiCD5+ phenotype controls T cell-dependent inflammatory responses. Immunity 28, 639-50 (2008).

54. Rosser, E. C. \& Mauri, C. Regulatory B cells: origin, phenotype, and function. Immunity 42, 607-12 (2015).

55. Zhang, Y. et al. Mammary-tumor-educated B cells acquire LAP/TGF- $\beta$ and PD-L1 expression and suppress anti-tumor immune responses. Int. Immunol. 28, 423-433 (2016).

56. Olkhanud, P. B. et al. Tumor-evoked regulatory B cells promote breast cancer metastasis by converting resting CD4+ $\mathrm{T}$ cells to T-regulatory cells. Cancer Res. 71, 3505-15 (2011).

57. Muller, A. J. et al. Chronic inflammation that facilitates tumor progression creates local immune suppression by inducing indoleamine 2,3 dioxygenase. Proc. Natl. Acad. Sci. U. S. A. 105, 17073-8 (2008).

58. Park, M.-J. et al. Myeloid-Derived Suppressor Cells Induce the Expansion of Regulatory B Cells and Ameliorate Autoimmunity in the Sanroque Mouse Model of Systemic Lupus Erythematosus. Arthritis Rheumatol. 68, 2717-2727 (2016).

59. Lelis, F. J. N. et al. Myeloid-derived suppressor cells modulate B-cell responses. Immunol. Lett. 188, 108-115 (2017).

60. Shen, M. et al. A novel MDSC-induced PD-1-PD-L1+ B-cell subset in breast tumor microenvironment possesses immuno-suppressive properties. Oncoimmunology 7 , e1413520 (2018).

61. Bodogai, M. et al. Immunosuppressive and Prometastatic Functions of Myeloid-Derived Suppressive Cells Rely upon Education from Tumor-Associated B Cells. Cancer Res. 75, 3456-3465 (2015).

62. Li, A. et al. Indoleamine 2,3-dioxygenase 1 inhibition targets anti-PD1-resistant lung tumors by blocking myeloid-derived suppressor cells. Cancer Lett. 431, 54-63 (2018).

63. Jitschin, R. et al. CLL-cells induce IDOhi CD14+HLA-DRlo myeloid-derived suppressor cells that inhibit T-cell responses and promote TRegs. Blood 124, 750-760 (2014).

64. Yu, J. et al. Myeloid-derived suppressor cells suppress antitumor immune responses through IDO expression and correlate with lymph node metastasis in patients with breast cancer. J. Immunol. 190, 3783-97 (2013).

65. Wang, Y. et al. Myeloid-Derived Suppressor Cells Impair B Cell Responses in Lung Cancer through IL-7 and STAT5. J. Immunol. 201, 278-295 (2018).

66. Trigunaite, A. et al. Gr-1 high CD11b+Cells Suppress B Cell Differentiation and Lupus-like Disease in Lupus-Prone Male Mice. Arthritis Rheum. 65, 2392-2402 (2013).

67. Danaher, P. et al. Gene expression markers of Tumor Infiltrating Leukocytes. J. Immunother. Cancer 5, (2017).

68. Tirosh, I. et al. Dissecting the multicellular ecosystem of metastatic melanoma by singlecell RNA-seq. Science (80-. ). 352, 189-196 (2016).

69. Sade-Feldman, M. et al. Defining T Cell States Associated with Response to Checkpoint 
Immunotherapy in Melanoma. Cell 175, 998-1013.e20 (2018).

70. Munn, D. H. et al. Prevention of allogeneic fetal rejection by tryptophan catabolism. Science 281, 1191-3 (1998).

71. Munn, D. H. et al. Potential regulatory function of human dendritic cells expressing indoleamine 2,3-dioxygenase. Science 297, 1867-70 (2002).

72. Uyttenhove, C. et al. Evidence for a tumoral immune resistance mechanism based on tryptophan degradation by indoleamine 2,3-dioxygenase. Nat. Med. 9, 1269-74 (2003).

73. Mehraj, V. \& Routy, J.-P. Tryptophan Catabolism in Chronic Viral Infections: Handling Uninvited Guests. Int. J. Tryptophan Res. 8, IJTR.S26862 (2015).

74. Ravishankar, B. et al. Tolerance to apoptotic cells is regulated by indoleamine 2,3dioxygenase. Proc. Natl. Acad. Sci. U. S. A. 109, 3909-3914 (2012).

75. Matteoli, G. et al. Gut CD103+ dendritic cells express indoleamine 2,3-dioxygenase which influences $\mathrm{T}$ regulatory/T effector cell balance and oral tolerance induction. Gut 59, 595-604 (2010).

76. Muller, A. J., DuHadaway, J. B., Donover, P. S., Sutanto-Ward, E. \& Prendergast, G. C. Inhibition of indoleamine 2,3-dioxygenase, an immunoregulatory target of the cancer suppression gene Bin1, potentiates cancer chemotherapy. Nat. Med. 11, 312-9 (2005).

77. Mitchell, T. C. et al. Epacadostat Plus Pembrolizumab in Patients With Advanced Solid Tumors: Phase I Results From a Multicenter, Open-Label Phase I/II Trial (ECHO202/KEYNOTE-037). J. Clin. Oncol. 36, 3223-3230 (2018).

78. Zakharia, Y. et al. Interim Analysis of the Phase 2 Clinical Trial of the IDO Pathway Inhibitor Indoximod in Combination With Pembrolizumab for Patients With Advanced Melanoma. Oral Present. 107th Annu. Meet. Am. Assoc. Cancer Res. Abstract C, (2017).

79. Li, M. et al. The indoleamine 2,3-dioxygenase pathway controls complement-dependent enhancement of chemo-radiation therapy against murine glioblastoma. J. Immunother. Cancer 2, (2014).

80. Beatty, G. L. et al. First-in-Human Phase I Study of the Oral Inhibitor of Indoleamine 2,3Dioxygenase-1 Epacadostat (INCB024360) in Patients with Advanced Solid Malignancies. Clin. Cancer Res. 23, 3269-3276 (2017).

81. Hollern, D. P. et al. B Cells and T Follicular Helper Cells Mediate Response to Checkpoint Inhibitors in High Mutation Burden Mouse Models of Breast Cancer. Cell 179, 1191-1206.e21 (2019).

82. Schmid, P. et al. Pembrolizumab for early triple-negative breast cancer. N. Engl. J. Med. 382, 810-821 (2020).

83. Long, G. V et al. Epacadostat plus pembrolizumab versus pembrolizumab alone in patients with unresectable or metastatic melanoma: Results of the phase $3 \mathrm{ECHO}$ 301/KEYNOTE-252 study. J. Clin. Oncol. 36, 108-108 (2018).

84. Scott, G. N. et al. The immunoregulatory enzyme IDO paradoxically drives B cellmediated autoimmunity. J. Immunol. 182, 7509-17 (2009).

85. Holmgaard, R. B. et al. Tumor-Expressed IDO Recruits and Activates MDSCs in a TregDependent Manner. Cell Rep. 13, 412-24 (2015).

86. Baban, B. et al. Indoleamine 2,3-dioxygenase expression is restricted to fetal trophoblast giant cells during murine gestation and is maternal genome specific. J. Reprod. Immunol. 61, 67-77 (2004).

87. Holmgaard, R. B. et al. Tumor-Expressed IDO Recruits and Activates MDSCs in a TregDependent Manner. Cell Rep. 13, 412-24 (2015). 